\title{
Applications of Jacobi's Elliptic Functions in Forced Vibration in Nonlinear Rotor Dynamics
}

Gulama-Garip Alisher Ibrayev ( $\square$ ybraev.alysher@mail.ru )

Al-Farabi Kazakh National University https://orcid.org/0000-0001-5000-0023

Isaac Elishakoff

Florida Atlantic University

Almatbek Kydyrbekuly

Al-Farabi Kazakh National University

\section{Research Article}

Keywords: Jacobi's Elliptic Functions, Nonlinear Rotor Dynamics, Forced Vibration, Duffing's equation, multidegree-of-freedom

Posted Date: June 14th, 2021

DOI: https://doi.org/10.21203/rs.3.rs-459341/v1

License: (c) (1) This work is licensed under a Creative Commons Attribution 4.0 International License. Read Full License 


\title{
Applications of Jacobi's Elliptic Functions in Forced Vibration in Nonlinear Rotor Dynamics
}

\author{
Gulama-Garip Alisher Ibrayev ${ }^{1}$, Isaac Elishakoff ${ }^{2}$, Almatbek Kydyrbekuly ${ }^{1}$ \\ ${ }^{1}$ Al-Farabi Kazakh National University, Kazakhstan \\ e-mail: ybraev.alysher@mail.ru \\ ${ }^{2}$ Florida Atlantic University, USA \\ e-mail: elishako@fau.edu
}

\begin{abstract}
In this paper studied a rotary system with a nonlinearity, the equations of motion of which is one of the types of the Duffing's equations with multidegree-of-freedom, and also consider the advantages of using the elliptic function method to solve problems of this type. The system studied in this paper is also distinguished by the fact that in addition to the rotor vibrations in elastic supports with a nonlinearity, the vibrations of the foundation are also taken into account. The article presents a comparative analysis of the numerical Runge-Kutta-Fehlberg's 4-order method with an error estimate of 5-order, the approximate analytical Van der Pol's method and the elliptic function method proposed by the authors by comparing the obtained equations of motion of the system, as well as by comparing the constructed frequency response characteristic. From the results obtained, it follows that the method proposed by the authors can serve as a more accurate, general case of previously used approximate methods.
\end{abstract}

Keywords Jacobi's Elliptic Functions, Nonlinear Rotor Dynamics, Forced Vibration, Duffing's equation, multidegree-of-freedom.

\section{Introduction}

Currently, technological equipment may consist of many high-speed, highprecision mechanisms, including a complex control system and rotating elements, i.e. rotor systems. Jeffcott [1], Stodola [2], Dimentberg [3], Kelson [4], Kramer [5], Tiwari. [6], Rao [7], Tondl [8], Adams [9], Yamamoto and Ishida [10], Muszynska [11], Genta [12] and others made contributions to the study of the dynamics of rotor systems. Their work formed the basis of those physical and mathematical representations that served the further development of this field. Focusing on various issues related to the peculiarities of rotor dynamics when taking into account various factors, we can distinguish the works of the following authors, which address individual issues, such as various methods for determining critical 
velocities, for example, using modal analysis [12]; construction of Campbell frequency diagrams for determining critical velocities taking into account gyroscopic moments $[13,14]$; conducting a complete analysis of rotor dynamics from determining critical speeds to obtaining the amplitudes of oscillations and orbits of the rotor motion under the influence of an imbalance in an ordinary and complex form [15]; studying the dynamics of rotors taking into account some features, for example, high-speed rotors with complex rotation [16]; ensuring the stability of movement of rigid and flexible rotors [17]; analysis of nonlinear oscillations of rotors caused by the presence of various factors, for example, the use of non-linear supports, which lead to the appearance of sub-- and super--harmonic oscillations [18, 19], self-oscillating modes [20], and other phenomena depending on the type of bearings used in the machine [21, 22].

To date, there are numerous studies of the nonlinear dynamics of rotor systems [23-30]. It must be emphasized that despite the widespread occurrence of such systems, their dynamics have not been sufficiently studied due to the difficulties associated with the need to consider the combined action of factors, such as the influence of a liquid during partial filling, external non-conservative forces, linear and angular imbalances.

In the design of rotary machines, one of the most important mechanical components described by nonlinear models that determines the operability and reliability of the system [24-28] are elastic bearings, in our case, rolling bearings act as elastic bearings. Ignoring the nonlinear properties of bearings alters the results of both qualitatively and quantitatively $[23,29,30]$. This is explained by the fact that when analyzing linear rotor systems with rolling bearings, an approximate estimate of the stiffness and damping properties of bearings is most often used, when in reality the stiffness of a bearing substantially depends on loading, i.e. from the operating mode of the rotor system, from the geometry and size of the clearance in the bearing, from the size of the fit of the inner and outer rings in the bearing, etc. $[31,32]$.

Bearing mathematical models that consider nonlinearity factors are distinguished by complexity and, first of all, by the loads that they consider. In many cases, the Hertz theory is used to describe the bearing model, which relates the radial loads acting on the bearing and the deformation at the points of contact between the rolling body and the bearing rings [11]. When describing the bearing 
model, it is assumed that there are no phenomena of slipping of bodies or rolling surfaces. Damping is usually incorporated in the formulation of equivalent viscous and linear friction [33]. Also, for the most complete description of the process, it is important to consider the influence a change in inertial parameters, imbalance and asymmetry of the rotor installation on the shaft, external friction, and various kinds of positional forces [33-35]. Such complications of the model when analyzing the dynamics make it possible to study the influence of the gap size, rotation frequency on the frequency spectra and amplitude-frequency characteristics for any rotor system on rolling bearings.

At the moment, the use of rolling bearings has many advantages compared to magnetic bearings and lubricated plain bearings. Rolling bearings are relatively simple to manufacture (the same plain bearings are more complicated to manufacture, moreover, the thickness of the oil film very much affects the dynamics of the rotor system. For example, when manufacturing it is worth considering that with an increase in the film thickness the amplitudes of self-oscillations also increase $[6,10,36]$, and insufficient film thickness will lead to a violation of the thermal regime in the bearing, which leads to quick wear of the stud), they are more durable, and do not require special working conditions as magnetic bearings. In most cases, the approximation by a cubic power series is used to describe the nonlinear properties of rolling bearings [9, 10, 38-40].

In this paper, we study the forced oscillations of a vertical rotor system, which is described by a system of inhomogeneous nonlinear (cubic) differential equations of the second order, which are known as the Duffing equations. The Duffing equation is one of the classical equations of nonlinear dynamics [41]. This equation describes a fairly wide range of phenomena of the world around us [42]. The equation was first studied by German engineer Georg Duffing in 1918 and accordingly named in his honor. A lot of scientists worked on solving the problems associated with Duffing equation. At the moment, there is exists no exact solution in of this equation.

The Duffing equation can conditionally be considered in certain cases as an equation with "weak" nonlinearity, and in other cases as an equation with "strong" nonlinearity. The case with "weak" nonlinearity allows approximate solutions of the equation, since as practice shows, the solution in this case is nearly periodic. In this regard, this case has been fairly well studied by such authors as Stoker [43], 
Rao [44], Nayfeh and Mook [47], Klotter [50], Timoshenko [49], Hayashi [45], Tondl [46], Magnus [51], Kauderer, Balachandran, Benaroya [48], Kelly. This case was solved with sufficient accuracy by various methods, namely by techniques of averaging, partial linearization, asymptotic, and graphical methods. For example, authors such as Stoker, Hayashi, Rao, on the assumption that oscillations occur relative to a stable state, solve this equation by substituting a periodic function [4345].

Authors such as Tondl and Schmidt [46] use a different approach to solving this problem. They use an approximate method of slowly varying amplitudes, where the amplitude of the oscillations is considered as a function of time. To analyze the stability of stationary solutions, linearized equations in variations are used.

Other authors such as Nayfeh, Mook, Balachandran, Benaroya [47] use various asymptotic methods to solve the perturbed Duffing equation. For example, Nayfeh, Mook and Balachandran used the so-called multi-scale method, the idea of which is to switch from given variables to slowly changing variables. Benaroya used methods of expansion in a small parameter to solve this problem [48].

It should also be noted that authors such as Timoshenko and Klotter used the Ritz averaging methods $[49,50]$, where it is assumed that an approximate solution of the steady-state oscillations can be represented as a series. Among the authors who obtained an approximate solution by the method of partial linearization, it should be noted Magnus who managed to obtain a relationship between the amplitude and frequency of the system by the harmonic balance method [51]. It should be noted that there are also exact solutions of the Duffing equation without damping, expressed through elliptic functions described by Cveticanin, Kovacic, Hsu [52, 53, 56] and Starossek [57]. Likewise, based upon the elliptic functions, some approximate methods were advanced, such as Poincare-Lindstedt and KrylovBogolyubov methods, generalized Galerkin method, and the multiple scales method.

\section{Problem Statement}

To construct the equations of motion of the system, a fixed coordinate system $O x y z$ is introduced (Figure 1). Let be that in the equilibrium state the geometric center of the shaft (rotor) and the center of gravity of the foundation coincides with the axis $O z$. The coordinates in the shifted position of the center of the shaft (rotor) $O_{1}$ are 
denoted by $x_{1}$ and $y_{1}$, and the coordinates of the center of gravity of the rotor are indicated by $x_{s}$ and $y_{s}$. The coordinates of the center of gravity $\mathrm{O}_{2}$ of the foundation are indicated by $x_{2}$ and $y_{2}$, respectively. It is postulated that the rotor undertakes a plane-parallel motion, and there is no rotation of the foundation around the coordinate axes.
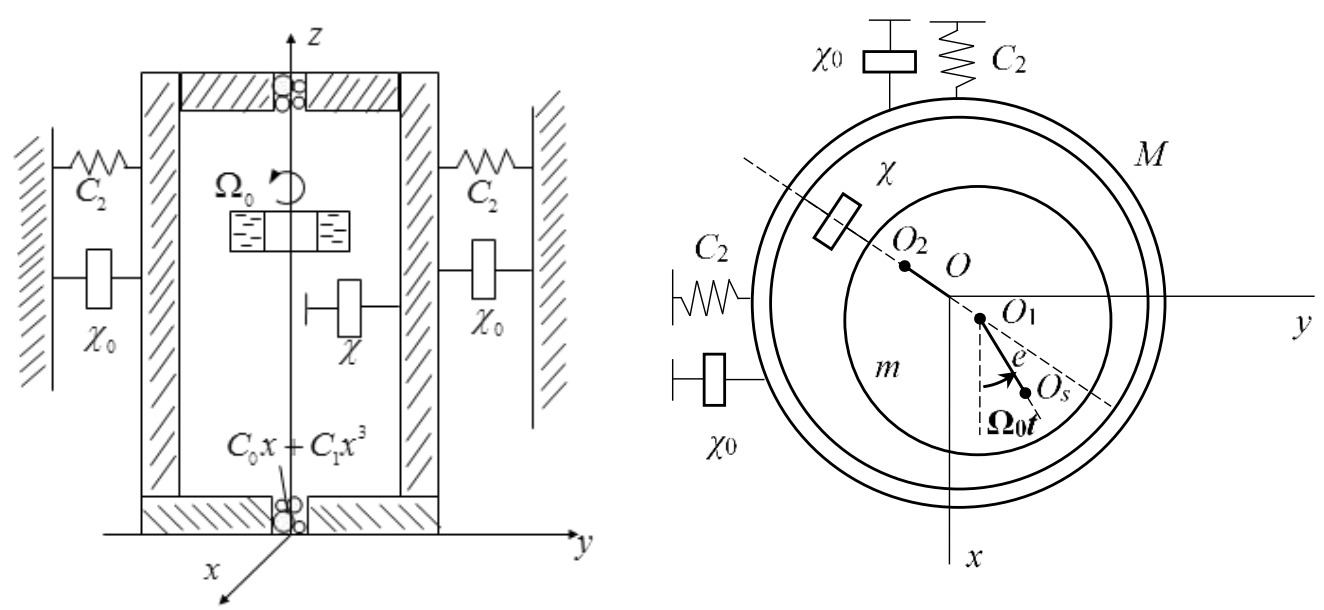

Fig. 1 Scheme of a rotor on rolling bearings

To derive the equations of motion, we use the following notation. Let $\Omega_{0}$ denote angular velocity of the rotor (shaft); radial force and radial deformation in a ball bearing which are fitted respectively by using the Hertzian contact formulation and cubic polynomial, and the specific expressions are given as follows: the Hertzian contact,

$$
F_{r}=C_{\mathrm{b}} \delta_{r}^{\frac{3}{2}}
$$

and the cubic polynomial,

$$
F_{r}=c_{1} \delta_{r}^{3}+c_{0} \delta_{r}
$$

where $F_{r}$ represents the radial force $(\mathrm{N}), \delta_{r}$ is the radial deformation $(\mathrm{m}), C_{\mathrm{b}}$ is the contact stiffness $\left(\mathrm{N} / \mathrm{m}^{3 / 2}\right), c_{0}$ and $c_{1}$ are respectively, the cubic and linear coefficients of the cubic polynomial. It can be found that the Hertzian analytical formulation is in good agreement with the experimental data when the maximum deformation is under $140 \mu \mathrm{m}$, which is not large enough. However, the possibility that these two models are equivalent is in a wide range of deformation. Thus, the predicted maximum deformation value is $1000 \mu \mathrm{m}$ based on the experimental Hertzian 
analytical formulation, and then the data are fitted by cubic polynomial [58]. In our case, we will call coefficients $c_{0}$ and $c_{1}$ are stiffness factors (or constants) of the rotor support (stiffness of the rolling bearing), $c_{2}-$ stiffness coefficient of the foundation support; $\chi$ and $\chi_{0}$ - external friction coefficients acting on the rotor and foundation. The kinetic energy of the rotor is determined by the formula:

$T_{1}=\frac{m}{2}\left(\dot{x}_{s}^{2}+\dot{y}_{s}^{2}\right)+\frac{J}{2} \Omega_{0}^{2}$

where $J$ - polar moment of inertia of the rotor, $m$ - mass of the rotor. The kinetic energy of the foundation is expressed by the formula:

$T_{2}=\frac{M}{2}\left(\dot{x}_{2}^{2}+\dot{y}_{2}^{2}\right)$

where $M$ is the mass of the foundation. The total kinetic energy of the system is given by

$T=T_{1}+T_{2}=\frac{m}{2}\left(\dot{x}_{s}^{2}+\dot{y}_{s}^{2}\right)+\frac{J}{2} \Omega_{0}^{2}+\frac{M}{2}\left(\dot{x}_{2}^{2}+\dot{y}_{2}^{2}\right)$.

The potential energy of the isotropic elastic field of a foundation support

$\Pi_{1}=\frac{c_{2}}{2}\left(x_{2}^{2}+y_{2}^{2}\right)$

Considering that

$\delta_{r}^{2}=\left(x_{1}-x_{2}\right)^{2}+\left(y_{1}-y_{2}\right)^{2}$

The potential energy of the isotropic nonlinear elastic field of the rolling bearing is determined by the formula

$\Pi_{2}=\frac{c_{0}}{2}\left[\left(x_{1}-x_{2}\right)^{2}+\left(y_{1}-y_{2}\right)^{2}\right]+\frac{c_{1}}{4}\left[\left(x_{1}-x_{2}\right)^{4}+\left(y_{1}-y_{2}\right)^{4}\right]+\ldots$.

We energy dissipation function reads

$\Phi=\frac{\chi}{2}\left(\dot{x}_{1}^{2}+\dot{y}_{1}^{2}\right)+\frac{\chi_{0}}{2}\left(\dot{x}_{2}^{2}+\dot{y}_{2}^{2}\right)$.

The coordinates of the center of gravity of the rotor are given as follows:

$x_{s}=x_{1}+e \cos \Omega_{0} t$,
$y_{s}=y_{1}+e \sin \Omega_{0} t$ 
where $e$ - the value of the rotor static imbalance (linear displacement of the rotor center of mass from its geometric center $-O_{1} O_{s}$ ).

The total potential energy of the system is:

$\Pi=\Pi_{1}+\Pi_{2}$

Now, using the Lagrange equations of the second kind, we write the equations of motion of the system:

$$
\begin{aligned}
& m \ddot{x}_{1}+c_{0}\left(x_{1}-x_{2}\right)+c_{1}\left(x_{1}-x_{2}\right)^{3}+\chi \dot{x}_{1}=m e \Omega_{0}{ }^{2} \cos \Omega_{0} t, \\
& m \ddot{y}_{1}+c_{0}\left(y_{1}-y_{2}\right)+c_{1}\left(y_{1}-y_{2}\right)^{3}+\chi \dot{y}_{1}=m e \Omega_{0}^{2} \sin \Omega_{0} t, \\
& M \ddot{x}_{2}+c_{2} x_{2}-c_{0}\left(x_{1}-x_{2}\right)-c_{1}\left(x_{1}-x_{2}\right)^{3}+\chi_{0} \dot{x}_{2}=0, \\
& M \ddot{y}_{2}+c_{2} y_{2}-c_{0}\left(y_{1}-y_{2}\right)-c_{1}\left(y_{1}-y_{2}\right)^{3}+\chi_{0} \dot{y}_{2}=0,
\end{aligned}
$$

with the following initial conditions

$$
\begin{aligned}
& x_{1}(0)=e, x_{2}(0)=0.1 \cdot e, y_{1}(0)=0, y_{2}(0)=0, \\
& \dot{x}_{1}(0)=0, \dot{x}_{2}(0)=0, \dot{y}_{1}(0)=0, \dot{y}_{2}(0)=0 .
\end{aligned}
$$

The system of equations (12) describes the coupled motion of an unbalanced rotor and foundation with non-linear supports. In the absence of foundation movement, system (12) will consist of two equations describing the equation of motion of the rotor in nonlinear supports, which have been studied in sufficient detail in the [54, $55]$.

We introduce the following dimensionless parameters

$$
x_{1}=e f_{1}, x_{2}=e f_{2}, y_{1}=e z_{1}, y_{2}=e z_{2}, \mu=\frac{m}{M}, \omega_{1}^{2}=\frac{c_{0}}{m}, \omega_{2}^{2}=\frac{c_{2}}{m}, \tau=\omega_{1} t, \Omega_{0}=\omega_{1} \eta
$$

Then the system of equations (12) we obtain

$$
\begin{aligned}
& \ddot{f}_{1}+2 \zeta_{1} \dot{f}_{1}+\left(f_{1}-f_{2}\right)+\varepsilon\left(f_{1}-f_{2}\right)^{3}=\eta^{2} \cos (\eta \tau), \\
& \ddot{z}_{1}+2 \zeta_{1} \dot{z}_{1}+\left(z_{1}-z_{2}\right)+\varepsilon\left(z_{1}-z_{2}\right)^{3}=\eta^{2} \sin (\eta \tau), \\
& \ddot{f}_{2}+2 \mu \zeta_{2} \dot{f}_{2}-\mu\left(f_{1}-f_{2}\right)-\mu \varepsilon\left(f_{1}-f_{2}\right)^{3}+\mu \lambda f_{2}=0, \\
& \ddot{z}_{2}+2 \mu \zeta_{2} \dot{z}_{2}-\mu\left(z_{1}-z_{2}\right)-\mu \varepsilon\left(z_{1}-z_{2}\right)^{3}+\mu \lambda z_{2}=0,
\end{aligned}
$$

where

$$
\zeta_{1}=\frac{\chi}{2 m \omega_{1}}, \zeta_{2}=\frac{\chi_{0}}{2 m \omega_{1}}, \varepsilon=\frac{c_{1} e^{2}}{m \omega_{1}^{2}}, \lambda=\frac{\omega_{2}^{2}}{\omega_{1}^{2}} .
$$


Consider the forced oscillations of the rotor and the foundation. Forced oscillations of the system are caused by rotor imbalance. With a harmonic disturbing force, after some transient process, periodic oscillations multiple of the frequency of the disturbing force (steady-state oscillations) are established. The first and third equations of system (15) are not related to the second and fourth equations and their solutions can be sought independently of each other. In the future we will use the solutions of the first and third equations. We write the first and third equations in the form:

$$
\begin{aligned}
& \ddot{f}_{1}+2 \zeta_{1} \dot{f}_{1}+\left(f_{1}-f_{2}\right)+\varepsilon\left(f_{1}-f_{2}\right)^{3}=\eta^{2} \cos (\eta \tau), \\
& \ddot{f}_{2}+2 \mu \zeta_{2} \dot{f}_{2}-\mu\left(f_{1}-f_{2}\right)-\mu \varepsilon\left(f_{1}-f_{2}\right)^{3}+\mu \lambda f_{2}=0 .
\end{aligned}
$$

\section{Classic Approximate Solution}

For the solve this problem and for the comparing the results that will be obtained using the elliptic function we use by classical averaged Van der Pol's method. To do this, we will find for the functions $f_{1}(\tau)$ and $f_{2}(\tau)$ in system (17) in the following form, i.e.

$$
\begin{aligned}
& f_{1}(\tau)=A_{1}(\tau) \cos (\eta \tau)+B_{1}(\tau) \sin (\eta \tau), \\
& f_{2}(\tau)=A_{2}(\tau) \cos (\eta \tau)+B_{2}(\tau) \sin (\eta \tau),
\end{aligned}
$$

where is $A_{1}(\tau), A_{2}(\tau), B_{1}(\tau)$ and $B_{2}(\tau)$ in slowly varying values for the period of the oscillations. Substituting (18) into the system of equations (17) and equating the left and right sides of the equations with the same functions, we obtain

$$
\begin{aligned}
& \ddot{A}_{1}-\eta^{2} A_{1}+2 \dot{B}_{1} \eta+2 \zeta_{1}\left(\dot{A}_{1}+B_{1} \eta\right)+\left(A_{1}-A_{2}\right)+\frac{3}{4} \varepsilon\left(\left(A_{1}-A_{2}\right)^{3}+\left(A_{1}-A_{2}\right)\left(B_{1}-B_{2}\right)^{2}\right)=\eta^{2}, \\
& \ddot{B}_{1}-\eta^{2} B_{1}-2 \dot{A}_{1} \eta+2 \zeta_{1}\left(\dot{B}_{1}-A_{1} \eta\right)+\left(B_{1}-B_{2}\right)+\frac{3}{4} \varepsilon\left(\left(B_{1}-B_{2}\right)^{3}+\left(A_{1}-A_{2}\right)^{2}\left(B_{1}-B_{2}\right)\right)=0, \\
& \ddot{A}_{2}-\eta^{2} A_{2}+2 \dot{B}_{2} \eta+2 \mu \zeta_{2}\left(\dot{A}_{2}+B_{2} \eta\right)-\mu\left(A_{1}-A_{2}\right)-\frac{3}{4} \mu \varepsilon\left(\left(A_{1}-A_{2}\right)^{3}+\left(A_{1}-A_{2}\right)\left(B_{1}-B_{2}\right)^{2}\right)+\mu \lambda A_{2}=0, \\
& \ddot{B}_{2}-\eta^{2} B_{2}-2 \dot{A}_{2} \eta+2 \mu \zeta_{2}\left(\dot{B}_{2}-A_{2} \eta\right)-\mu\left(B_{1}-B_{2}\right)-\frac{3}{4} \mu \varepsilon\left(\left(B_{1}-B_{2}\right)^{3}+\left(A_{1}-A_{2}\right)^{2}\left(B_{1}-B_{2}\right)\right)+\mu \lambda B_{2}=0 .
\end{aligned}
$$

For stationarity of solutions to system (19), it is necessary that $A_{1}(\tau), A_{2}(\tau), B_{1}(\tau)$ and $B_{2}(\tau)$ be constant, then 


$$
\begin{aligned}
& \left(1-\eta^{2}\right) A_{1}-A_{2}+2 \eta \zeta_{1} B_{1}+\frac{3}{4} \varepsilon\left(\left(A_{1}-A_{2}\right)^{3}+\left(A_{1}-A_{2}\right)\left(B_{1}-B_{2}\right)^{2}\right)=\eta^{2} \\
& \left(1-\eta^{2}\right) B_{1}-B_{2}+2 \eta \zeta_{1} A_{1}+\frac{3}{4} \varepsilon\left(\left(B_{1}-B_{2}\right)^{3}+\left(A_{1}-A_{2}\right)^{2}\left(B_{1}-B_{2}\right)\right)=0 \\
& -\mu A_{1}+\left(\mu \lambda-\eta^{2}+\mu\right) A_{2}+2 \mu \eta \zeta_{2} B_{2}-\frac{3}{4} \mu \varepsilon\left(\left(A_{1}-A_{2}\right)^{3}+\left(A_{1}-A_{2}\right)\left(B_{1}-B_{2}\right)^{2}\right)=0 \\
& -\mu B_{1}+\left(\mu \lambda-\eta^{2}+\mu\right) B_{2}+2 \mu \eta \zeta_{2} A_{2}-\frac{3}{4} \mu \varepsilon\left(\left(B_{1}-B_{2}\right)^{3}+\left(A_{1}-A_{2}\right)^{2}\left(B_{1}-B_{2}\right)\right)=0
\end{aligned}
$$

The amplitudes obtained from the system (20) are shown in Figures 3 and 4.

\section{Elliptic functions: basic relationships}

Since elliptic functions are a generalized case of trigonometric functions. The trigonometric functions can be geometrically represented using a circle that describes the trajectory of the center of mass of a linear oscillator. The motion of a nonlinear oscillator can be interpreted as the motion of the center of mass of the rotor $m$ (in case of foundation $M$ ) by ellipse (Fig. 2). Hence, geometrically, elliptic functions can be represented as

$$
\operatorname{cn}(u, k)=\frac{x}{a}, \operatorname{sn}(u, k)=\frac{y}{b}=y, \operatorname{dn}(u, k)=\frac{r}{a},
$$

where $b=1$, and

$$
u=\int_{A}^{B} r d \varphi=\int_{A}^{B} \frac{d \varphi}{\sqrt{1-k^{2} \sin ^{2} \varphi}}, k=\sqrt{1-\frac{1}{a^{2}}} .
$$

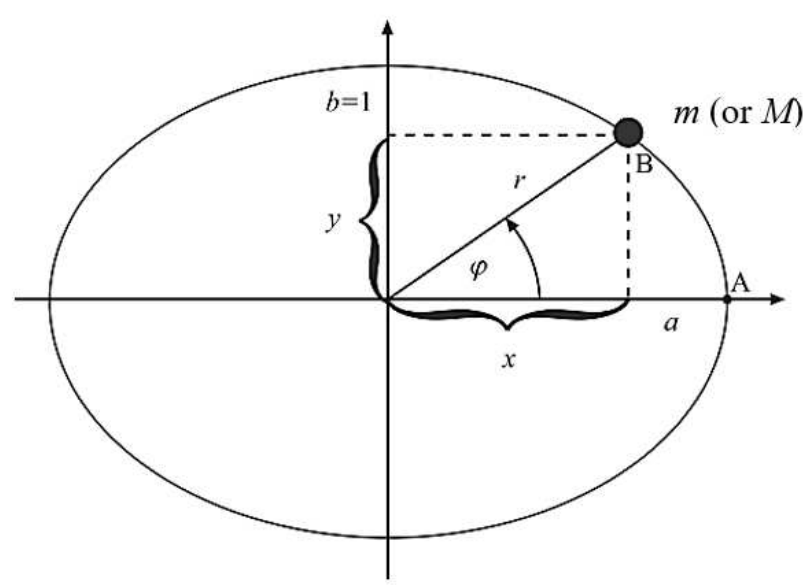

Fig. 2 Interpretation the motion of the center of mass of the rotor and foundation

where 
$\varphi=\operatorname{amp}(u, k)=\int_{0}^{u} \operatorname{dn}\left(u^{\prime}, k\right) d u^{\prime}$,

The function $\operatorname{amp}(u)$ is the inverse of the elliptic integral of the first kind, i.e.:

$u=F(\varphi, k)=\int_{0}^{\varphi} \frac{d \xi}{\sqrt{1-k^{2} \sin ^{2} \xi}}$

The main properties of elliptic functions are

$$
\begin{aligned}
& \operatorname{cn}^{2}(u, k)+\operatorname{sn}^{2}(u, k)=1, \operatorname{dn}^{2}(u, k)+k^{2} \operatorname{sn}^{2}(u, k)=1, \operatorname{dn}(u, k)=\sqrt{1-k^{2} \operatorname{sn}^{2}(u, k)} \\
& \frac{d}{d u} \operatorname{sn}(u, k)=\operatorname{cn}(u, k) \operatorname{dn}(u, k), \frac{d}{d u} \operatorname{cn}(u, k)=-\operatorname{sn}(u, k) \operatorname{dn}(u, k), \\
& \frac{d}{d u} \operatorname{dn}(u, k)=-k^{2} \operatorname{sn}(u, k) \operatorname{cn}(u, k) .
\end{aligned}
$$

\section{Application of Elliptic Functions for the Solution}

To solve system (17), we assume that that is the perturbing force $\cos (\eta \tau)$ there is a special case of the disturbing force of the form $\operatorname{cn}\left(\omega_{f} \tau, k\right)$, that is, we assume that $\eta$ $\equiv \omega_{f}$, and when $k=0, \operatorname{cn}\left(\omega_{f} \tau, 0\right)=\cos (\eta \tau)$. Given this statement, we rewrite the system (17) in the following general form

$$
\begin{aligned}
& \ddot{f}_{1}+2 \zeta_{1} \dot{f}_{1}+\left(f_{1}-f_{2}\right)+\varepsilon\left(f_{1}-f_{2}\right)^{3}=\omega_{f}^{2} \operatorname{cn}\left(\omega_{f} \tau, k\right), \\
& \ddot{f}_{2}+2 \mu \zeta_{2} \dot{f}_{2}-\mu\left(f_{1}-f_{2}\right)-\mu \varepsilon\left(f_{1}-f_{2}\right)^{3}+\mu \lambda f_{2}=0 .
\end{aligned}
$$

The solution of system (26) will be found in the form

$$
\begin{aligned}
& f_{1}=A_{1} \operatorname{cn}\left(\omega_{f} \tau, k\right)+B_{1} \operatorname{sn}\left(\omega_{f} \tau, k\right), \\
& f_{2}=A_{2} \operatorname{cn}\left(\omega_{f} \tau, k\right)+B_{2} \operatorname{sn}\left(\omega_{f} \tau, k\right),
\end{aligned}
$$

where $k$ - elliptic modulus, $\omega_{f}$ - circular frequency of disturbing force, $\operatorname{cn}\left(\omega_{f} \tau, k\right)$ and $\operatorname{sn}\left(\omega_{f} \tau, k\right)$ - elliptical cosine and sine.

Considering basic relationships of elliptic functions (25), we obtain

$$
\begin{aligned}
& \frac{d f_{i}}{d \tau}=\frac{d f_{i}}{d u} \cdot \frac{d u}{d \tau}=-\omega_{f} A_{i} \mathrm{sn} \mathrm{dn}+\omega_{f} B_{i} \mathrm{cndn}, \\
& \frac{d^{2} f_{i}}{d \tau^{2}}=-\omega_{f}^{2} A_{i}\left(1-2 k^{2} \mathrm{sn}^{2}\right) \mathrm{cn}-\omega_{f}^{2} k^{2} B_{i}\left(1-k^{2}+2 k^{2} \mathrm{cn}^{2}\right) \mathrm{sn},
\end{aligned}
$$

where 
$\mathrm{cn} \equiv \mathrm{cn}\left(\omega_{f} \tau, k\right), \operatorname{sn} \equiv \operatorname{sn}\left(\omega_{f} \tau, k\right), \operatorname{dn} \equiv \operatorname{dn}\left(\omega_{f} \tau, k\right), i=1,2$

Substituting the solution in the form (27) into the system (26) and taking into account (28), we get

$$
\begin{aligned}
\operatorname{cn} & {\left[\omega_{f}^{2}\left(2 k^{2}-1\right) A_{1}+\left(A_{1}-A_{2}\right)+3 \varepsilon\left(A_{1}-A_{2}\right)\left(B_{1}-B_{2}\right)^{2}-\omega_{f}^{2}\right]+} \\
& +\operatorname{sn}\left[\omega_{f}^{2}\left(k^{2}-1\right) B_{1}+\left(B_{1}-B_{2}\right)+\varepsilon\left(B_{1}-B_{2}\right)^{3}\right]+ \\
& +\mathrm{cn}^{3}\left[\varepsilon\left(A_{1}-A_{2}\right)^{3}-2 \omega_{f}^{2} k^{2} A_{1}-3 \varepsilon\left(A_{1}-A_{2}\right)\left(B_{1}-B_{2}\right)^{2}\right]+ \\
& +\operatorname{sncn}^{2}\left[\varepsilon\left(3\left(A_{1}-A_{2}\right)^{2}-\left(B_{1}-B_{2}\right)^{2}\right)\left(B_{1}-B_{2}\right)-2 \omega_{f}^{2} k^{2} B_{1}\right]+ \\
& +2 \zeta_{1} \omega_{f} B_{1} \mathrm{cndn}-2 \zeta_{1} \omega_{f} A_{1} \operatorname{sndn}=0, \\
\operatorname{cn} & {\left[\omega_{f}^{2}\left(2 k^{2}-1\right) A_{2}-\mu\left(A_{1}-A_{2}\right)-3 \mu \varepsilon\left(A_{1}-A_{2}\right)\left(B_{1}-B_{2}\right)^{2}+\mu \lambda A_{2}\right]+} \\
& +\operatorname{sn}\left[\omega_{f}^{2}\left(k^{2}-1\right) B_{2}-\mu\left(B_{1}-B_{2}\right)-\mu \varepsilon\left(B_{1}-B_{2}\right)^{3}+\mu \lambda B_{2}\right]+ \\
& +\operatorname{cn}^{3}\left[-\mu \varepsilon\left(A_{1}-A_{2}\right)^{3}-2 \omega_{f}^{2} k^{2} A_{2}+3 \mu \varepsilon\left(A_{1}-A_{2}\right)\left(B_{1}-B_{2}\right)^{2}\right]+ \\
& +\operatorname{sncn}^{2}\left[-\mu \varepsilon\left(3\left(A_{1}-A_{2}\right)^{2}-\left(B_{1}-B_{2}\right)^{2}\right)\left(B_{1}-B_{2}\right)-2 \omega_{f}^{2} k^{2} B_{2}\right]+ \\
& +2 \mu \zeta_{2} \omega_{f} B_{2} \mathrm{cndn}-2 \mu \zeta_{2} \omega_{f} A_{2} \operatorname{sndn}=0 .
\end{aligned}
$$

or

$$
\begin{aligned}
& \operatorname{cn}\left[\omega_{f}^{2}\left(2 k^{2}-1\right) A_{1}+\left(A_{1}-A_{2}\right)+3 \varepsilon\left(A_{1}-A_{2}\right)\left(B_{1}-B_{2}\right)^{2}-\omega_{f}^{2}+2 \zeta_{1} \omega_{f} B_{1} H_{2}\right]+ \\
& +\operatorname{sn}\left[\begin{array}{c}
\omega_{f}^{2}\left(k^{2}-1\right) B_{1}+\left(B_{1}-B_{2}\right)+\varepsilon\left(B_{1}-B_{2}\right)^{3}- \\
-2 \zeta_{1} \omega_{f} A_{1} H_{1}+H_{3}\left(\varepsilon\left(3\left(A_{1}-A_{2}\right)^{2}-\left(B_{1}-B_{2}\right)^{2}\right)\left(B_{1}-B_{2}\right)-2 \omega_{f}^{2} k^{2} B_{1}\right)
\end{array}\right]+ \\
& +\mathrm{cn}^{3}\left[\varepsilon\left(A_{1}-A_{2}\right)^{3}-2 \omega_{f}^{2} k^{2} A_{1}-3 \varepsilon\left(A_{1}-A_{2}\right)\left(B_{1}-B_{2}\right)^{2}\right]=0 \text {, } \\
& \operatorname{cn}\left[\omega_{f}^{2}\left(2 k^{2}-1\right) A_{2}-\mu\left(A_{1}-A_{2}\right)-3 \mu \varepsilon\left(A_{1}-A_{2}\right)\left(B_{1}-B_{2}\right)^{2}+\mu \lambda A_{2}+2 \mu \zeta_{2} \omega_{f} B_{2} H_{2}\right]+ \\
& +\operatorname{sn}\left[\begin{array}{c}
\omega_{f}^{2}\left(k^{2}-1\right) B_{2}-\mu\left(B_{1}-B_{2}\right)-\mu \varepsilon\left(B_{1}-B_{2}\right)^{3}+\mu \lambda B_{2}- \\
-2 \mu \zeta_{2} \omega_{f} A_{2} H_{1}+H_{3}\left(-\mu \varepsilon\left(3\left(A_{1}-A_{2}\right)^{2}-\left(B_{1}-B_{2}\right)^{2}\right)\left(B_{1}-B_{2}\right)-2 \omega_{f}^{2} k^{2} B_{2}\right)
\end{array}\right]+ \\
& +\mathrm{cn}^{3}\left[-\mu \varepsilon\left(A_{1}-A_{2}\right)^{3}-2 \omega_{f}^{2} k^{2} A_{2}+3 \mu \varepsilon\left(A_{1}-A_{2}\right)\left(B_{1}-B_{2}\right)^{2}\right]=0 \text {. }
\end{aligned}
$$

where is

$$
H_{1}=\frac{1}{\pi} \int_{0}^{2 \pi} \operatorname{sn} u \operatorname{dn} u \sin \varphi d \varphi=\frac{1}{\pi} \int_{0}^{4 K} \operatorname{sn}^{2} u \operatorname{dn}^{2} u d u=\frac{4}{3 k^{2} \pi}\left[\left(2 k^{2}-1\right) E+K\left(1-k^{2}\right)\right]
$$


$H_{2}=\frac{1}{\pi} \int_{0}^{2 \pi} \operatorname{cn} u \operatorname{dn} u \cos \varphi d \varphi=\frac{1}{\pi} \int_{0}^{4 K} \operatorname{cn}^{2} u \operatorname{dn}^{2} u d u=\frac{4}{3 k^{2} \pi}\left[\left(1+k^{2}\right) E-K\left(1-k^{2}\right)\right]$,

$H_{3}=\frac{1}{\pi} \int_{0}^{2 \pi} \operatorname{sn} u \operatorname{cn}^{2} u \sin \varphi d \varphi=\frac{1}{\pi} \int_{0}^{4 K} \operatorname{sn}^{2} u \operatorname{cn}^{2} u \operatorname{dn} u d u=\frac{1}{4}$.

Given these relations we obtain a system of algebraic equations

$$
\begin{aligned}
& \left(A_{1}-A_{2}\right)+\frac{1}{2} \omega_{f}^{2} k^{2} A_{1}-\omega_{f}^{2} A_{1}+\frac{3}{4} \varepsilon\left(A_{1}-A_{2}\right)\left(\left(A_{1}-A_{2}\right)^{2}+\left(B_{1}-B_{2}\right)^{2}\right)-\omega_{f}^{2}+2 \zeta_{1} \omega_{f} B_{1} H_{2}=0, \\
& \left(B_{1}-B_{2}\right)+\frac{1}{2} \omega_{f}^{2} k^{2} B_{1}-\omega_{f}^{2} B_{1}+\frac{3}{4} \varepsilon\left(B_{1}-B_{2}\right)\left(\left(A_{1}-A_{2}\right)^{2}+\left(B_{1}-B_{2}\right)^{2}\right)-2 \zeta_{1} \omega_{f} A_{1} H_{1}=0 \\
& \varepsilon\left(A_{1}-A_{2}\right)^{3}-2 \omega_{f}^{2} k^{2} A_{1}-3 \varepsilon\left(A_{1}-A_{2}\right)\left(B_{1}-B_{2}\right)^{2}=0 \\
& \mu\left((\lambda+1) A_{2}-A_{1}\right)+\frac{1}{2} \omega_{f}^{2} k^{2} A_{2}-\omega_{f}^{2} A_{2}-\frac{3}{4} \mu \varepsilon\left(A_{1}-A_{2}\right)\left(\left(A_{1}-A_{2}\right)^{2}+\left(B_{1}-B_{2}\right)^{2}\right)+2 \mu \zeta_{2} \omega_{f} B_{2} H_{2}=0, \\
& \mu\left((\lambda+1) B_{2}-B_{1}\right)+\frac{1}{2} \omega_{f}^{2} k^{2} B_{2}-\omega_{f}^{2} B_{2}-\frac{3}{4} \mu \varepsilon\left(B_{1}-B_{2}\right)\left(\left(A_{1}-A_{2}\right)^{2}+\left(B_{1}-B_{2}\right)^{2}\right)-2 \mu \zeta_{2} \omega_{f} A_{2} H_{1}=0 \\
& -\mu \varepsilon\left(A_{1}-A_{2}\right)^{3}-2 \omega_{f}^{2} k^{2} A_{2}+3 \mu \varepsilon\left(A_{1}-A_{2}\right)\left(B_{1}-B_{2}\right)^{2}=0 \\
& k^{2}=\frac{\varepsilon\left(A_{1}-A_{2}\right)\left(\left(A_{1}-A_{2}\right)^{2}-3\left(B_{1}-B_{2}\right)^{2}\right)}{2 \omega_{f}^{2} A_{1}} .
\end{aligned}
$$

where, changing the values $\omega_{f}$ you can build the responses characteristics of the vibrations of the rotor and the foundation.

The $E \equiv E(\varphi, k)$ the incomplete elliptic integral of the second kind, and $K \equiv K(\varphi, k)$ being the complete elliptic integral of the first kind is

$$
\begin{aligned}
& E(\varphi, k)=\int_{0}^{\varphi} \sqrt{1-k^{2} \sin ^{2} \varphi} d \varphi, \\
& K(\varphi, k)=\int_{0}^{\varphi} \frac{d \varphi}{\sqrt{1-k^{2} \sin ^{2} \varphi}} .
\end{aligned}
$$

For calculations, $K$ and $E$ can be expanded in a power series in $k$ as

$$
\begin{aligned}
& K=\frac{\pi}{2}\left[1+\frac{1}{4} k^{2}+\frac{9}{64} k^{4}+O\left(k^{6}\right)\right], \\
& E=\frac{\pi}{2}\left[1-\frac{1}{4} k^{2}-\frac{3}{64} k^{4}+O\left(k^{6}\right)\right] .
\end{aligned}
$$




\section{Results}

To verify the results obtained by the elliptic function method, along with equations (33) and (34), were obtained and compared the results of expanding the elliptic cosine in a series (equation (36)), the method of expansion in a Van der Pol's method (equation (20)), and numerical calculation with Runge-Kutta-Fehlberg's method of the system (15) when $e=0.001 \mathrm{~m}, m=25 \mathrm{~kg}, M=25 \mathrm{~kg}, \chi=4200 \mathrm{~kg} / \mathrm{s}$, $\chi_{0}=6.59 \mathrm{~kg} / \mathrm{s}, c_{2}=3.26 \cdot 10^{5} \mathrm{~kg} / \mathrm{s}^{2}, c_{1}=0.87 \cdot 10^{11} \mathrm{~kg} / \mathrm{m}^{2} \mathrm{~s}^{2}, c_{0}=1.1 \cdot 10^{7} \mathrm{~kg} / \mathrm{s}^{2}$ (Figures 3 and 4).

The two resonances are observed in the system, that are caused by vibration of the foundation. The first linear resonance appears at $\omega_{f}=0.051$ in all three cases both for the rotor and for the foundation. At the numerical calculation, the amplitudes of the first resonance are $f_{1}=3.75$ for the rotor and $f_{2}=3.74$ for the foundation. In the case of calculation by the Van der Pol's method accordingly $f_{1}=$ 7.7 and $f_{2}=3.4$, i.e. the deviation from results of the numerical calculation of the amplitude of the rotor is $105.3 \%$ and of the foundation is $9.1 \%$. By using the method of elliptic functions the amplitudes of the first resonance are close to the results of the numerical method and are for the rotor $-f_{1}=5$, for the foundation $-f_{2}=3.5$, meanwhile in this case the deviation of amplitudes of the rotor is $33.3 \%$, and of the foundation is $6.4 \%$ (Figures 3 and 4 ).

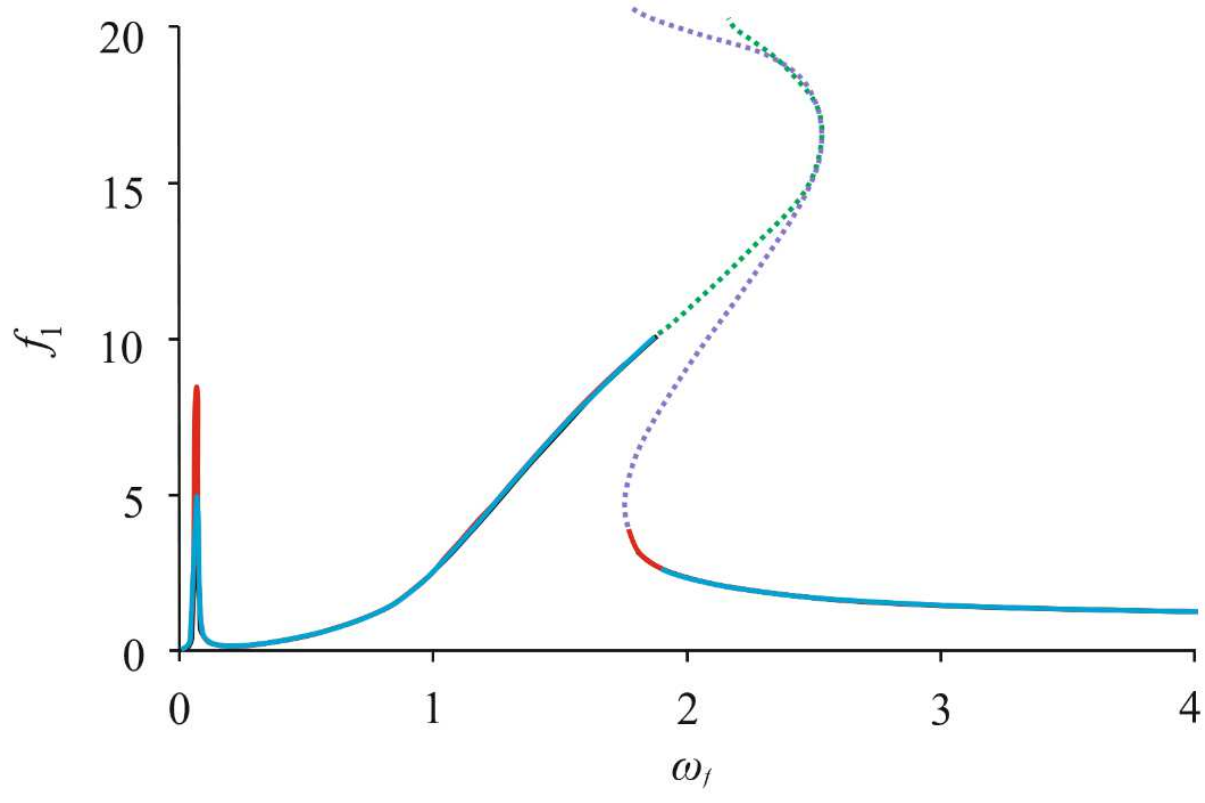

- Numerical Method • Van der Pol's Method • E EF Method

Fig. 3 Frequency response of the rotor with different methods 


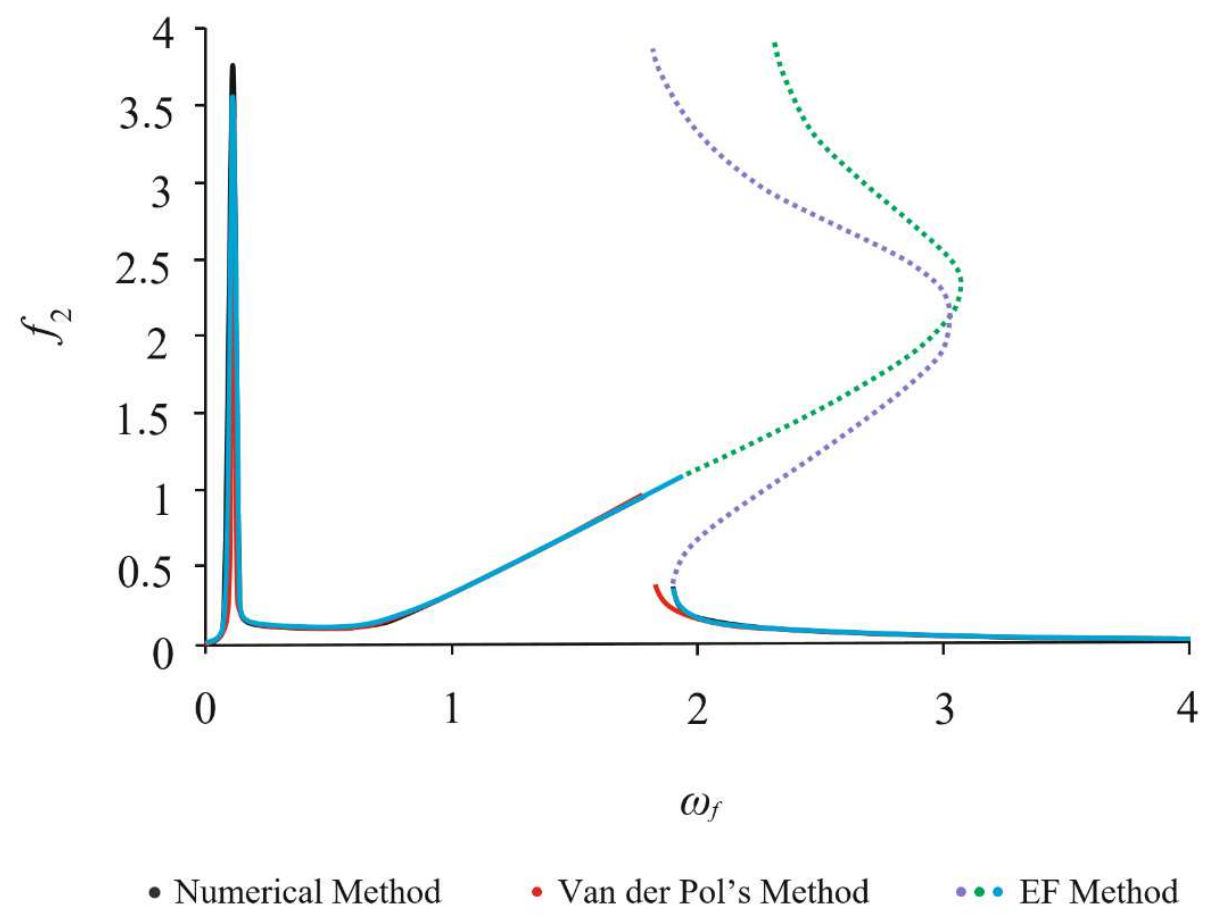

Fig. 4 Frequency response of the foundation using different methods

In the case of application each method at different frequencies the breakdown of the amplitudes is observed, that belongs to the second of the nonlinear resonance. In the case of application the Runge-Kutta-Fehlberg's method this breakdown occurs at $\omega_{f}=1.33$, while at the Van der Pol's method the breakdown is observed at $\omega_{f}=1.25$. In the case of application the method of elliptic functions the breakdown already occurs at $\omega_{f}=1.3$, that practically coincides with the results of the numerical method.

In this case, one of the main disadvantages of the numerical method is impossibility of calculation the maximum value of the amplitude of the nonlinear resonance. Here the difficulty lies in the fact that the maximum value of the amplitude of the nonlinear resonance is reached after the breakdown of the amplitudes. As for application the Van der Pol's method, it does not provide sufficient accuracy under certain conditions. Applying the method of elliptic functions eliminates these disadvantages due to generality and higher accuracy.

The determination of the maximum value of the amplitude and the design the amplitude-frequency and phase characteristics were achieved by many authors, among them Van der Pol, J.J.Stoker, A.H.Nayfeh, D.Mook, K.Klotter, S.P.Timoshenko, S.Hayashi, A.Tondl, K.Magnus, H.Kauderer, S.S.Rao, B.Balachandran, L.Cveticanin, I.Kovacic and so on. The methods, that were 
suggested by different groups of authors, in general can be divided into approximate analytical (the method of harmonic balance, the method of slowly varying amplitudes, the multiple scales and so on) and the graph analytical methods (the method of isoclines, the method of Lienar, the delta-method and so on).

J.J.Stoker, S.Hayashi, S.S.Rao, assuming that vibration occur relatively a stable equilibrium position, solved the nonlinear differential equation with cubic nonlinearity (the Duffing's equation) by the method of substitution with the assumption that the system carries out the harmonic vibration [43, 44, 45]. Van der Pol and A.Tondl proposed the approximate analytical method of slowly varying amplitudes, where besides assumption that the system carries out the harmonic vibration, the amplitude of vibration was considered as a function of time. On this occasion the linearized equations in variations were used to analysis the stability of stationary solutions [46]. A.H.Nayfeh, D.Mook and B.Balachandran applied the asymptotic method of many scales, where the terms, generating the secular terms, were eliminated by the asymptotic expansion of variable and derivative of the Poincare's type, i.e. by the degree of parameter, characterizing the nonlinearity [47, 42]. Among the many authors K.Magnus can be distinguished, who suggested the solution of this problem applying the method of harmonic balance, when the restoring force is linearized and the stiffness coefficient of that is a function of the amplitude [51]. Other authors, such as C.S.Hsu, L.Cveticanin, I.Kovacic, when searching for a solution to the nonlinear equation of motion, considered the more generalized method based on the application of Jacobi's elliptic functions, as a result of that the authors got the generalized system of equations, which degenerated with the ellipticity modulus equals to zero into the system of algebraic equations, that were got earlier by other authors $[52,53,56]$. In all of the above mentioned cases the maximum value of the amplitude, lying behind the field of the breakdown of the amplitudes, was found by superimposition the amplitude-frequency characteristics, obtained from the system of algebraic equations and excluding unstable stationary solutions.

S.P.Timoshenko, K.Klotter, D.H.Young and W.Weaver Jr., who applied the averaging Ritz's method for solution the equation of motion, where it is assumed that possible work carried out in one cycle is zero, they suggested a graphical method to calculate this maximum value; this method allows us to determine the maximum value of the amplitude by intersection of the family of hyperbolas, 
corresponding to the fundamental resonance frequency $\left(\omega_{f}=1\right)$ with the curve of the amplitude-frequency characteristic of free vibration of the relevant system [49, 50].

In our case the method of harmonic balance was applied in conjunction with the Jacobi's elliptic functions, that makes it possible to get the generalized system of algebraic equations of the amplitudes, which depend on the ellipticity modulus and that in its turn provide more accurate values of the amplitudes breakdown frequency and their maximum value (blue, green and purple dotted curves, Figures 3 and 4). Here the maximum values of the amplitudes of the rotor and the foundation are got by the intersection of unstable values (the dotted curves in green and purple colours), which can be obtained by solving the algebraic system of equations (the remaining curves corresponding to the multiple of the unstable values are not shown in Figures 3 and 4, in order that there is not cluttering image). Further, in the figures below, the dotted curves will be substituted by solid curves.

For assessing the maximum values of the amplitudes of the system and determining the optimal operating mode the amplitude-frequency characteristics of the rotor and the foundation were built at different degree of the nonlinearity $(\varepsilon)$, at different values of the damping coefficients $\left(\zeta_{1}\right.$ and $\left.\zeta_{2}\right)$, at different values of ratios of the linear stiffness coefficients $(\lambda)$, and at different values of the ratio of the masses of the rotor and the foundation $(\mu)$.

Decreasing the value of the nonlinearity (cases $\varepsilon=0.1$ and $\varepsilon=0.5$, Figures 5 and 6) for both the rotor and the foundation leads to increasing the value of the amplitude of the first linear resonance, while its frequency in both cases coincides with case $\varepsilon=1$, i.e. $\omega_{f}=0.051$. At $\varepsilon=0.5$ the amplitude of the rotor is $f_{1}=8$, and the amplitude of the foundation is $f_{2}=3.95$, that is 1.5 and 1.13 times larger than the case of $\varepsilon=1$ respectively. At $\varepsilon=0.1$ the amplitude of the rotor is $f_{1}=9.8$, and the amplitude of the foundation is $f_{2}=5$, that is already 1.96 and 1.43 times more than the case $\varepsilon=1$ respectively. At decreasing the nonlinearity of the amplitudes value of the second resonance do not change their value, while the frequency of the breakdown of the amplitudes shifts in the direction of decreasing of the frequencies, respectively, in this case the slope of the amplitude-frequency characteristic curve at the nonlinear resonance is declined, degenerating into the linear case. For instance, at $\varepsilon=0.5$ the breakdown is observed at $\omega_{f}=1.2$, whereas at $\varepsilon=0.1$ the breakdown of the amplitudes already occurs at $\omega_{f}=1.15$. Increasing the nonlinearity 
(cases $\varepsilon=5$ and $\varepsilon=10$, Figures 5 and 6) leads to decreasing the amplitudes value of the first linear resonance of the rotor and the foundation. In this case the frequency of the first resonance does not change and coincides with the case $\varepsilon=1$. At $\varepsilon=5$ the amplitude of the rotor is $f_{1}=3.95$, and the amplitude of the foundation is $f_{2}=3.25$, that is 1.26 and 1.08 times less than the case $\varepsilon=1$. At $\varepsilon=10$ the amplitude of the rotor is $f_{1}=2.2$, and the amplitude of the foundation is $f_{2}=2.5$, that is 2.27 and 1.4 times more than the case $\varepsilon=1$ respectively. At increasing the nonlinearity the values of the amplitudes of the second resonance, as well as at decreasing do not change their value. In this case the frequency of the breakdown of the amplitudes is shifted in the direction of the increasing of the frequencies. For example, at $\varepsilon=5$ the breakdown is observed at $\omega_{f}=1.4$, while at $\varepsilon=10$ the breakdown of the amplitudes already occurs at $\omega_{f}=1.43$. The slope of the amplitude-frequency characteristic curve at the nonlinear resonance increases, taking the form corresponding to the expressed hard nonlinearity.

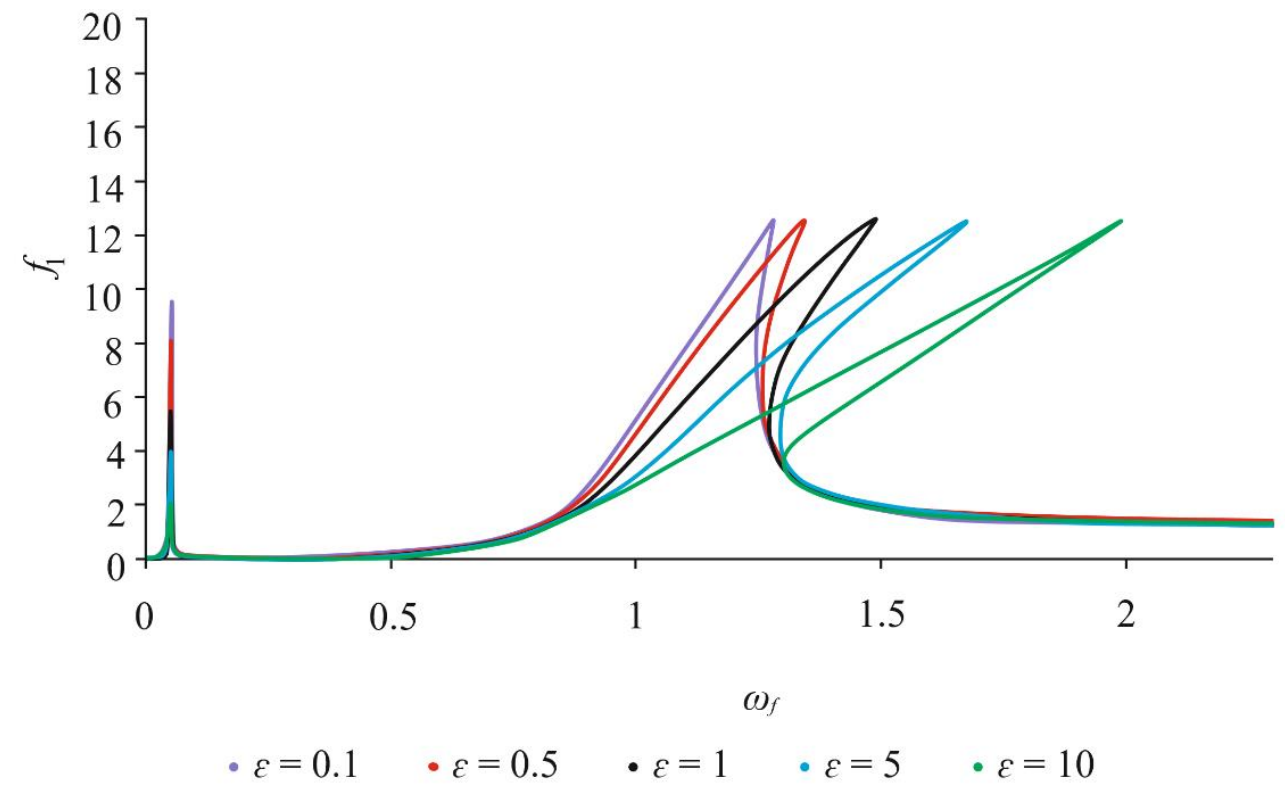

Fig. 5 Frequency response of the rotor with different coefficients of nonlinearity 


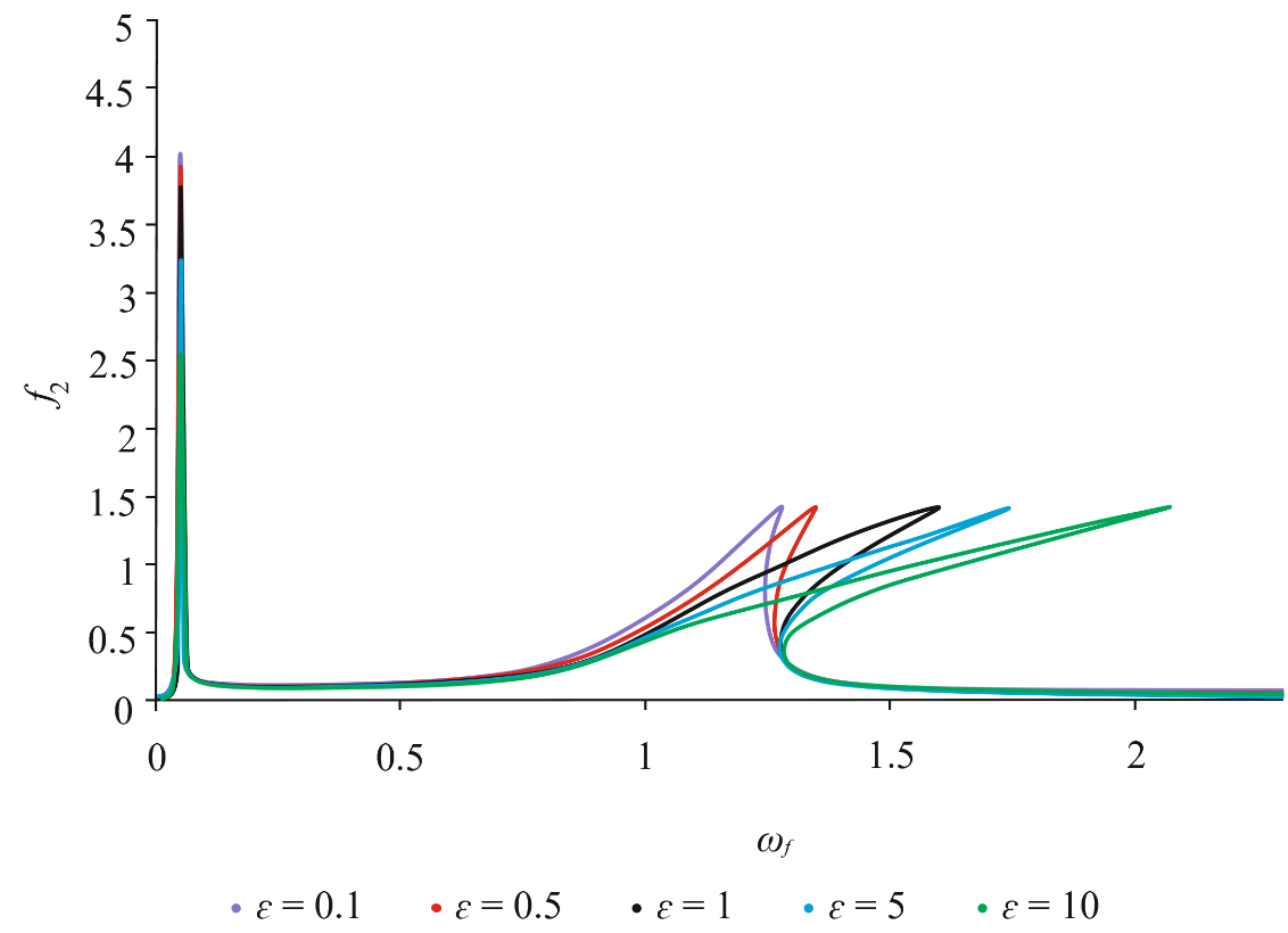

Fig. 6 Frequency response of the foundation with different coefficients of nonlinearity

The decreasing the damping coefficient (cases $\zeta_{1}=0.1$ and $\zeta_{2}=0.1, \zeta_{1}=0.5$ and $\zeta_{2}=0.5$, Figures 7 and 8) leads to growing of the amplitudes of both the first and the second resonance of the rotor and the foundation. At decreasing the damping coefficient by two times (case $\zeta_{1}=0.5$ and $\zeta_{2}=0.5$ ) the amplitudes of the rotor and the foundation increase by 1.5 times, i.e. at the first resonance $f_{1}=7.5, f_{2}$ $=5.25$, and at the second resonance $f_{1}=14, f_{2}=1.75$. At decreasing the damping coefficient by one order of value (case $\zeta_{1}=0.1$ and $\zeta_{2}=0.1$ ) the amplitudes of the rotor and the foundation increase by 2.25 times, i.e. at the first resonance $f_{1}=11.25$, $f_{2}=7.87$ and at the second one $f_{1}=15, f_{2}=2$. Increasing the damping coefficient (cases $\zeta_{1}=5$ and $\zeta_{2}=5, \zeta_{1}=10$ and $\zeta_{2}=10$, Figure 7 and 8) leads to decreasing the amplitudes of the first and the second resonances of the rotor and the foundation. For instance, at rising the damping coefficient by five times (case $\zeta_{1}=5$ and $\zeta_{2}=5$ ) the amplitudes of the rotor and the foundation decrease by 1.5 times, i.e. at the first resonance $f_{1}=3.33, f_{2}=2.33$, and at the second one $f_{1}=12, f_{2}=1.25$. At increasing the damping coefficient by one order of value, i.e. $\zeta_{1}=10$ and $\zeta_{2}=10$ the amplitudes of the rotor and the foundation decrease by 2.15 times, i.e. at the first resonance $f_{1}$ $=2.325, f_{2}=1.63$, and at the second one $f_{1}=10.05, f_{2}=0.95$. In these cases the resonance frequencies and the frequencies of the breakdown of the amplitudes do 
not change their values, i.e. the frequency of the first resonance and the breakdown of the amplitudes, as before are $\omega_{f}=0.051$ and $\omega_{f}=1.32$ respectively.

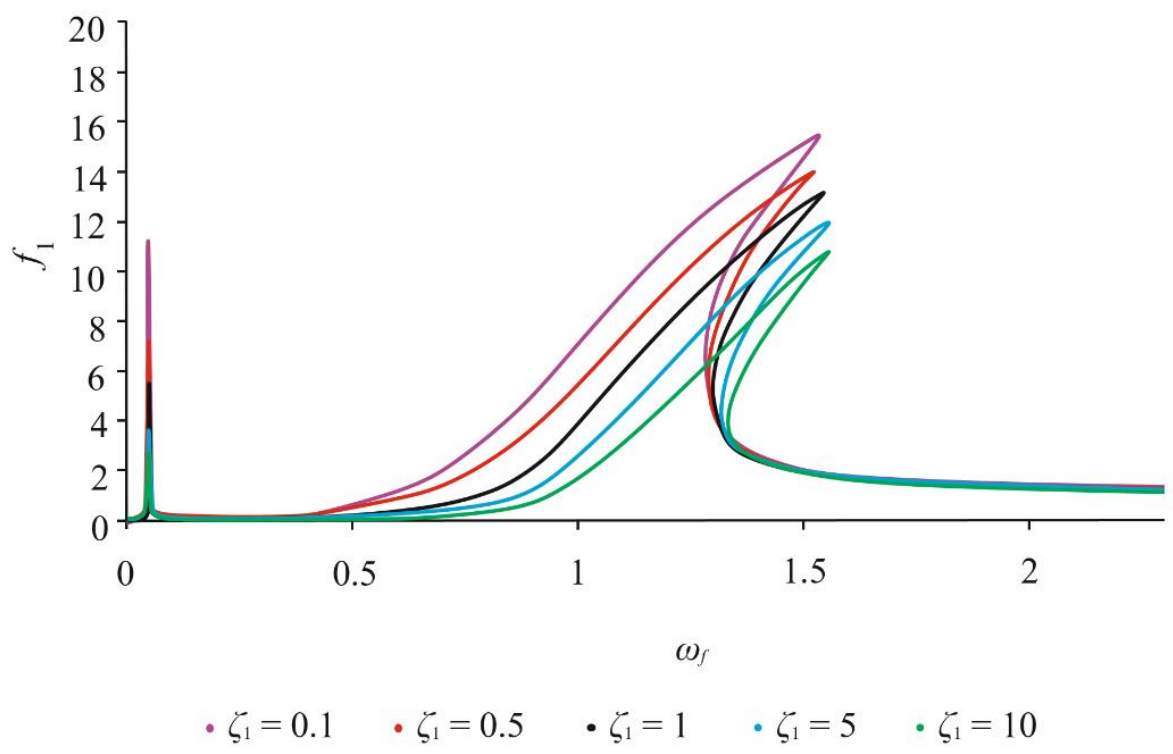

Fig. 7 Frequency response of the rotor with different damping ratio

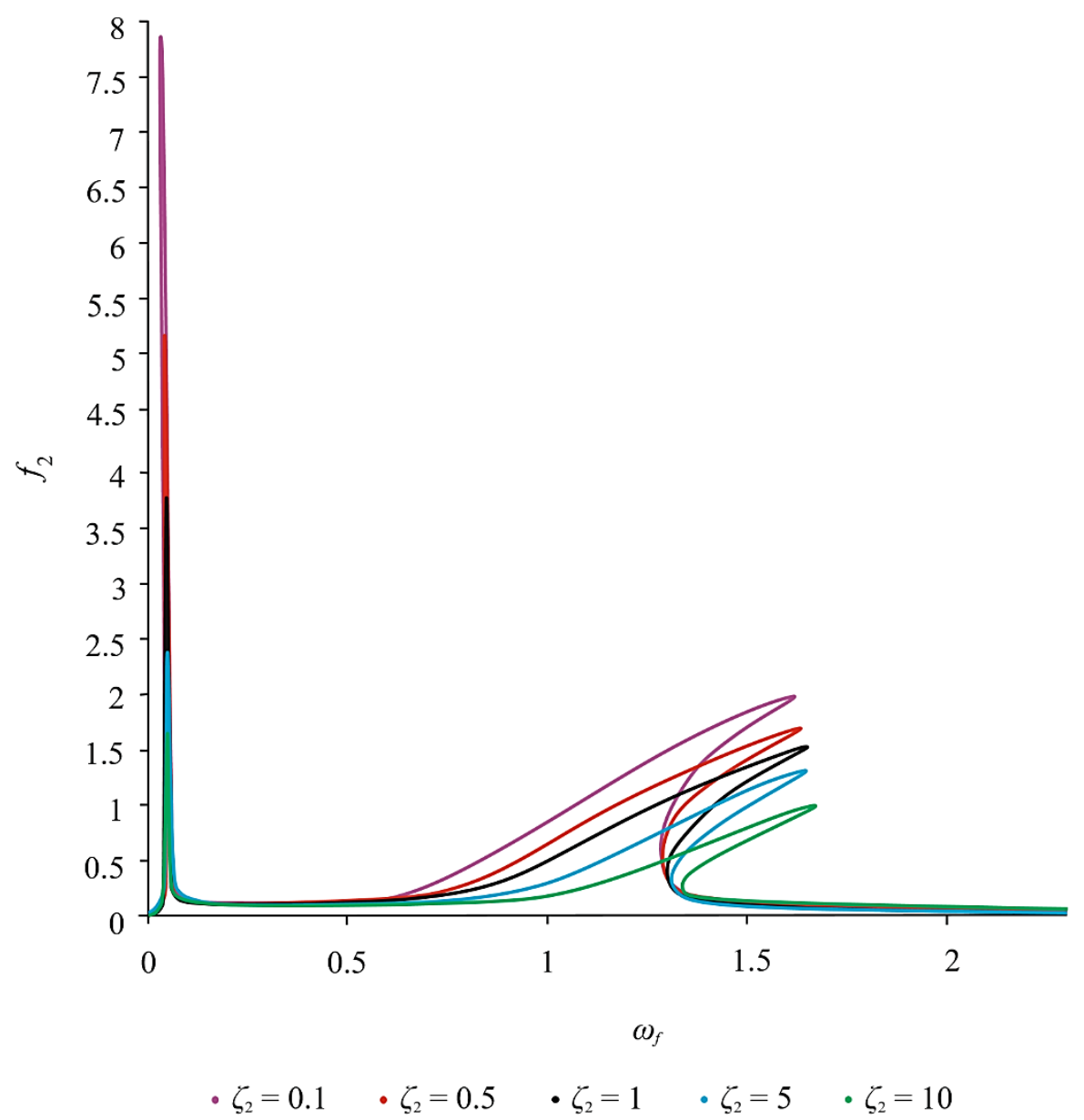

Fig. 8 Frequency response of the foundation with different damping ratio 
Increasing the ratio of the masses of the rotor and the foundation (cases $\mu=10$ and $\mu=5$, Figure 9 and 10) on account of decreasing the mass of the foundation leads to growing the amplitudes of the first and the second resonances of both the rotor and the foundation. At rising the ratio of the masses by five times (case $\mu=0.5$ ) the amplitude of the rotor is $f_{1}=7$, and the amplitude of the foundation is $f_{2}=3.9$, i.e. in this case the amplitude of the rotor increases by 1.4 times and the amplitude of the foundation by 1.1 times. At increasing the ratio of the masses by one order of value (case $\mu=10$ ) the amplitudes of the rotor and the foundation grow by 2.25 times, i.e. $f_{1}=11.25, f_{2}=7.87$. Decreasing the ratio of the masses (cases $\mu=0.5$ and $\mu=0.1$, Figures 9 and 10) as a whole leads to decreasing in the values of the amplitudes, that is due to the growing in the mass of the foundation, as in this case the foundation acts as an antiload. For example, at $\mu=0.5$ the amplitudes of the rotor and the foundation will be $f_{1}=4.7, f_{2}=2.75$, that is 1.06 and 1.27 times less than the case $\mu=1$ respectively. With a further decreasing the ratio of the masses, i.e. at $\mu=0.1 f_{1}=2.5, f_{2}=2$ that is already 2 and 1.97 times less than the case $\mu=$ 1.

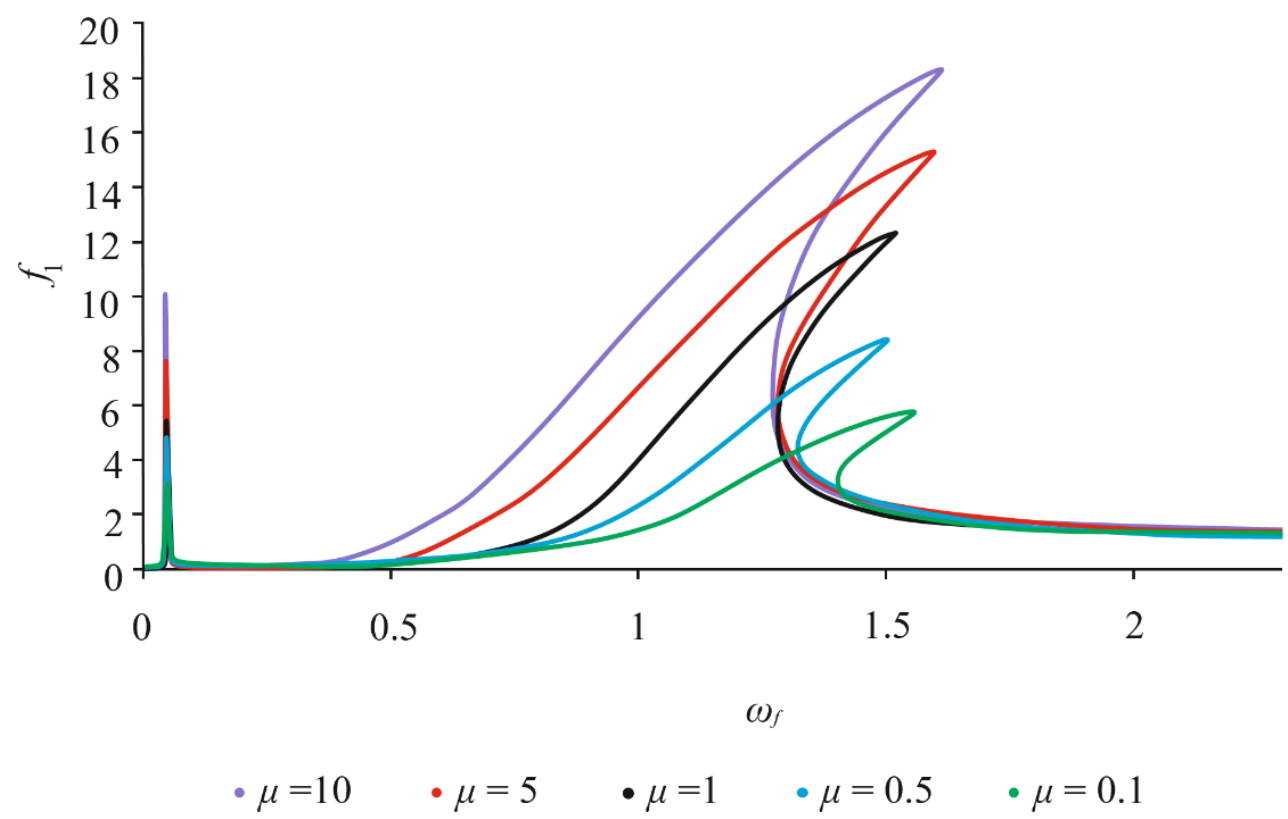

Fig. 9 Frequency response of the rotor with different mass ratio 


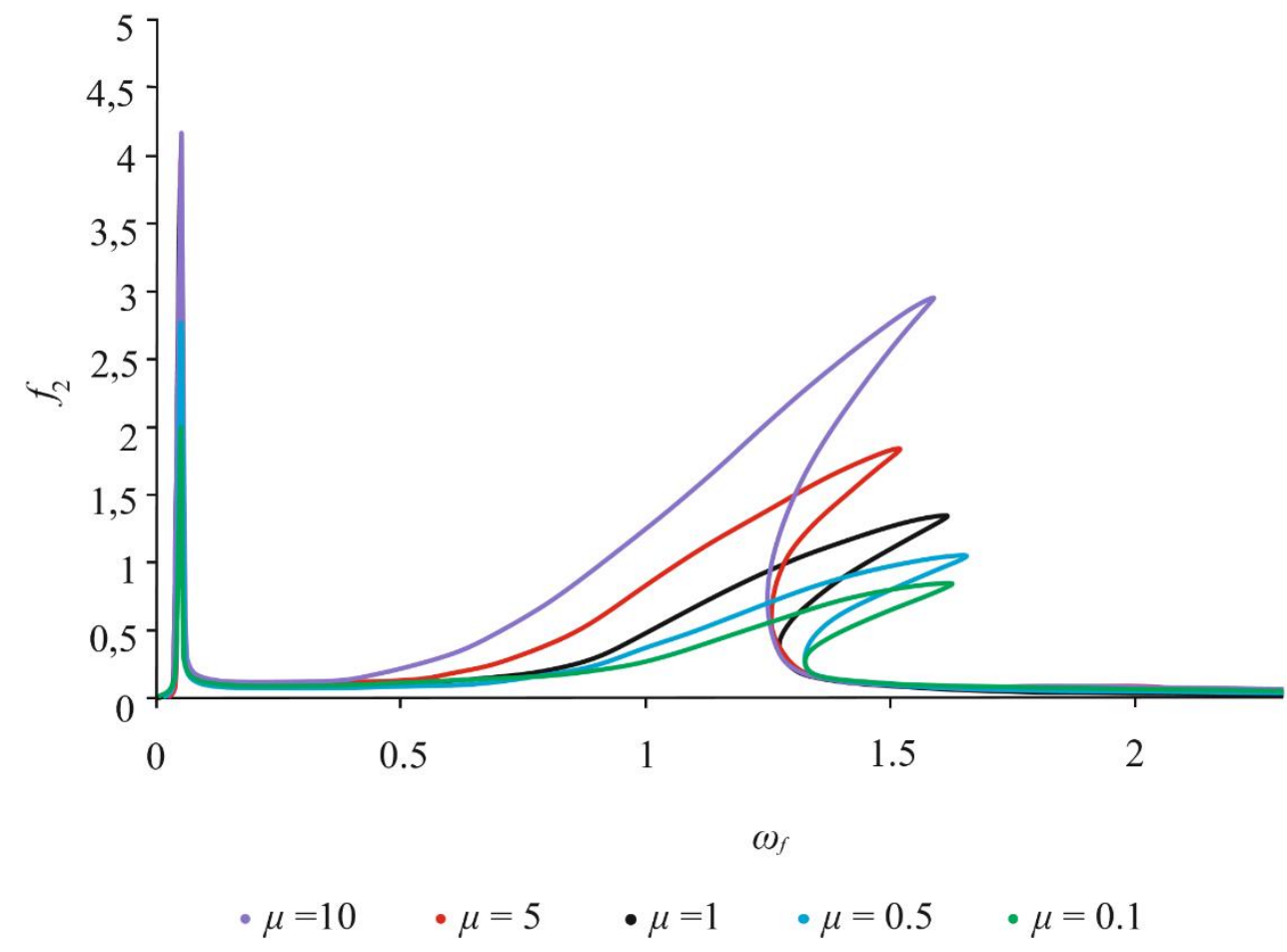

Fig. 10 Frequency response of the foundation with different mass ratio

The change in the ratio of the linear stiffness coefficients $\lambda$ leads to both a shift in the resonances and changes of the amplitudes in the value. For instance, when it decreases (cases $\lambda=0.1$ and $\lambda=0.5$, Figures 11 and 12), i.e. at increasing the linear stiffness coefficient $c_{0}$ the shift of the first and the second resonances along the frequencies axis towards its decreasing is observed, as well the growing the values of the amplitudes. At the decreasing the ratio of the linear stiffness coefficients by two times, i.e. at $\lambda=0.5$ the first linear resonance occurs at $\omega_{f}=0.04$, the breakdown of the amplitudes is already observed at $\omega_{f}=1.25$, while the values of the amplitudes will be $f_{1}=7.14, f_{2}=4.08$. When the ratios of the linear stiffness coefficients declines by one order of the value, i.e. at $\lambda=0.1$ the first linear resonance occurs at $\omega_{f}=0.04$, in this case the breakdown of the amplitudes is already observed at $\omega_{f}=1.15$ and the values of the amplitudes are $f_{1}=9.61, f_{2}=$ 7.8. Increasing of this parameter, i.e. declining of stiffness coefficient $c_{0}$, leads to the decreasing of the values of the amplitudes and to the shift of the first and the second resonances in the direction of the growing frequencies. For instance, when it is increased by five times (case $\lambda=5$ ) the first linear resonance occurs at $\omega_{f}=0.06$ and the breakdown of the amplitudes is observed at $\omega_{f}=1.4$ respectively, the values of the amplitudes will be $f_{1}=3.5, f_{2}=3$. In the case of the further increasing the 
ratio of the linear stiffness coefficients (case $\lambda=10$ ) the first linear resonance is already observed at $\omega_{f}=0.07$, and the breakdown of the amplitudes occurs at $\omega_{f}=$ 1.45. The values of the amplitudes of the rotor and the foundation will be $f_{1}=2.6$, $f_{2}=2$.

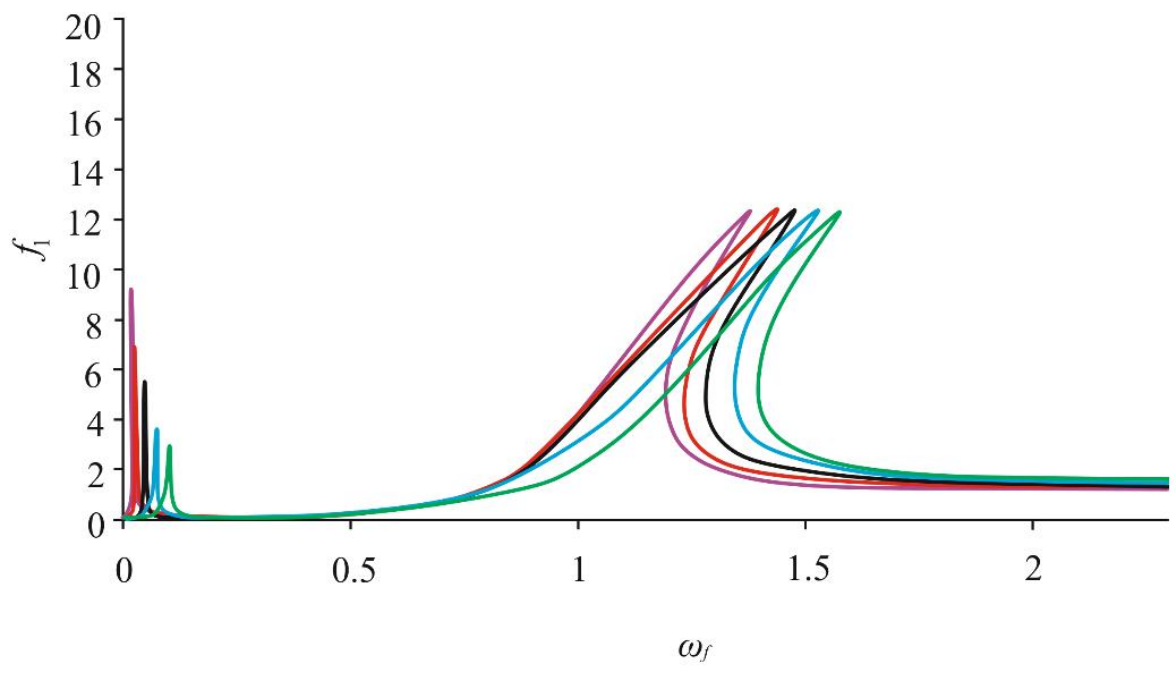

$$
\cdot \lambda=0.1 \quad \cdot \lambda=0.5 \quad \cdot \lambda=1 \quad \cdot \lambda=5 \quad \bullet \lambda=10
$$

Fig. 11 Frequency response of the rotor with different stiffness ratio

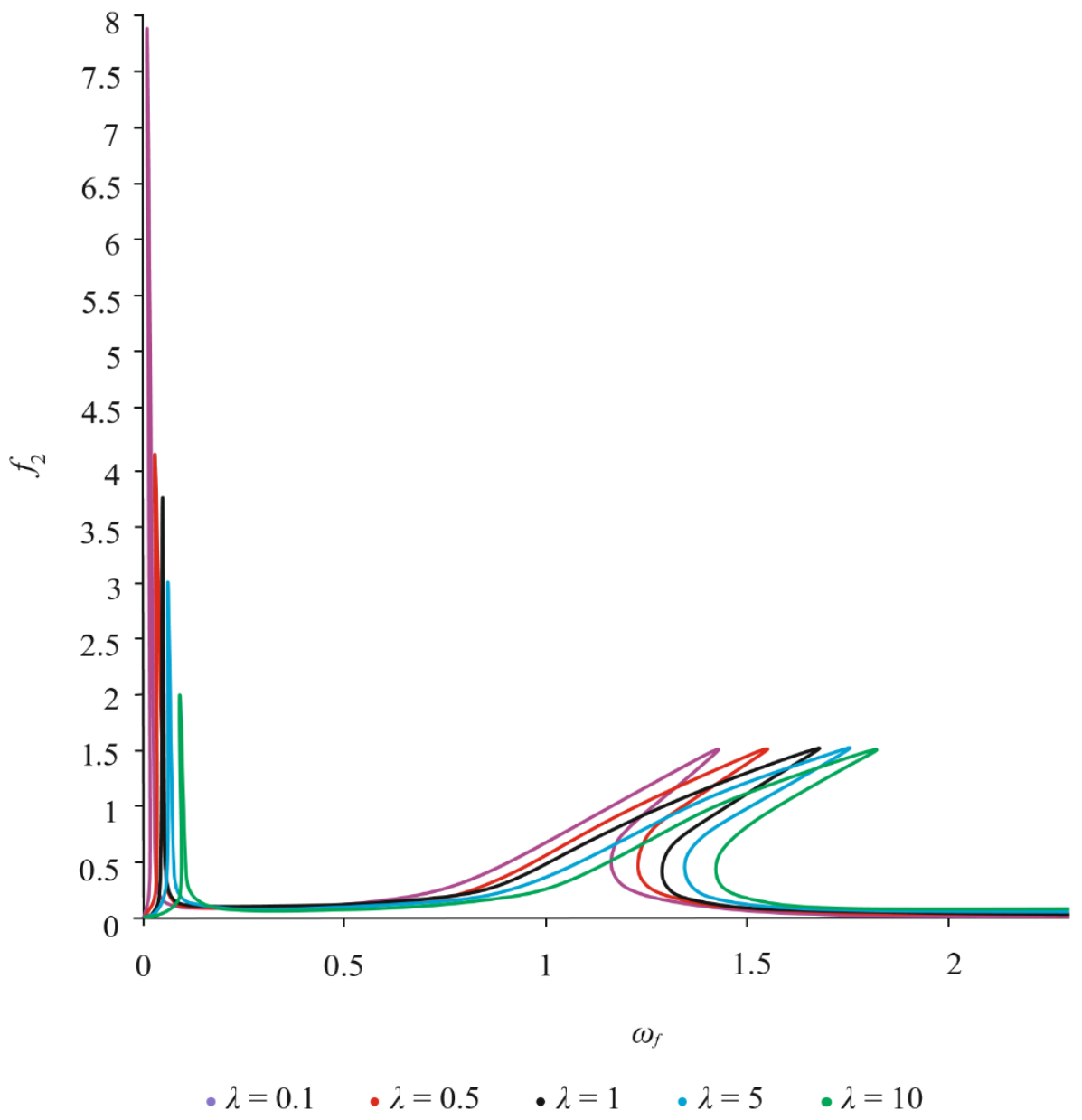

Fig. 12 Frequency response of the foundation with different stiffness ratio 


\section{Conclusion}

A method is developed for calculating the maximum value of the amplitudes and constructing the frequency characteristics of forced vibrations of the "rotorfoundation" system on rolling bearings with a nonlinear characteristic based on Jacobi's elliptic functions. The optimal parameters associated with the mass of the foundation, the coefficients of linear and nonlinear stiffness, as well as the damping coefficients, at which the magnitudes of the amplitudes have optimal values, have been determined. The features of the linear and nonlinear behavior of a system with many degrees of freedom, caused by vibrations of the foundation, are shown.

\section{Data availability statement}

Data sharing not applicable to this article as no datasets were generated or analysed during the current study.

Acknowledgements This work has been supported financially by the research project AP08856167 of the Ministry of Education and Science of the Republic of Kazakhstan and was performed at Research Institute of Mathematics and Mechanics in Al-Farabi Kazakh National University, which is gratefully acknowledged by the authors.

\section{Declarations}

Conflict of interest The authors declare that they have no conflict of interest.

\section{References}

1. Jeffcott, H. H.: The Lateral Vibration of Loaded Shafts in the neighborhood of a whirling speed. - The effect of want of balance. The London, Edinburgh, and Dublin Philosophical Magazine and Journal of Science, 37(219), 304-314 (1919)

2. Stodola, A.: Steam and Gas Tribunes. Authorized translation from the 6th German edition: Löwenstein LC. McGraw-Hill, New York (1927)

3. Dimentberg, F. M.: Flexural vibrations of rotating shafts. Butterworths, London (1961)

4. Kel'zon, A. S., Tsimanskii, Yu. P., Yakovlev, V.I.: Dynamics of Rotors in Elastic Supports. In Russian, Nauka, Moscow (1982) (in Russian)

5. Krämer, E.: Dynamics of rotors and foundations. Springer Science \& Business Media (2013)

6. Tiwari R.: Rotor systems: analysis and identification, Boca Raton: CRC Press (2018)

7. Rao, J.: History of Rotating Machinery Dynamics, Springer Science and Business Media B.V. (2011)

8. Tondl, A.: Some problems of rotor dynamics. Chapman and Hall, London (1965) 
9. Adams, M.L.: Rotating machinery vibration. New York: Marcel Dekker (2001)

10. Yamamoto, T., Ishida, Y.: Linear and nonlinear rotordynamics. New York: John Willey and Sons (2001)

11. Muszynska, A.: Rotordynamics. Boca Raton: Taylor\&Francis (2005)

12. Genta, G.: Dynamic Modelling of Rotors: A Modal Approach. IUTAM Symposium on Emerging Trends in Rotor Dynamics (pp. 27-38) (2011)

13. Campbell, W.: Protection of Steam Turbine Disk Wheels from Axial Vibration, Transactions of the ASME, 46, 31-160 (1924)

14. Lee, C.-W.: Evolution of Frequency-Speed Diagram in Rotating Machinery. IUTAM Symposium on Emerging Trends in Rotor Dynamics (pp. 39-50) (2009)

15. Greenhill, L.M., Cornejo G.A.: Critical Speeds Resulting from Unbalance Excitation of Backward Whirl Modes. Design Engineering Technical Conferences (DETC'95), 84(2), (pp. 991-1000) (1995)

16. Arakere, N., Schmitz, T., Cheng, C.-H.: Rotor Dynamic Response of a High-Speed Machine Tool Spindle. 23rd International Modal Analysis Conference (IMAC XXIII), 30, (2005)

17. Lesaffre, N., Sinou, J.-J., Thouverez, F.: Model and Stability Analysis of a Flexible Bladed Rotor, International Journal of Rotating Machinery, 2006, 1-16 (2006)

18. Ehrich, F.: Observations of Nonlinear Phenomena in Rotordynamics, Journal of System Design and Dynamics, 2(3), 641-651 (2008)

19. Pasynkova, I.A.: Dynamics of the precession motion of an unbalanced rotor: author. dis. for a job. scientific step. Dr. Phys.-Math. Sciences, St. Petersburg (2007) (in Russian)

20. Avramov, K. V.: Asymptotic analysis of forced vibrations of a one-disc rotor on a non-linear flexible base. Proceedings of the IMechE, Part C: Journal of Mech. Engineering.Science 224, 1593-1604 (2010)

21.Leontiev, M.K., Karasev, V.A., Potapova, O.Yu., Degtyarev, S.A., Leontiev, M.K.: The dynamics of the rotor in rolling bearings. Vibration of machines: measurement, reduction, protection. 4(7), 40-45 (2006)

22. Taranenko, P.A.: Dynamics of a rotor of a turbocompressor on sliding bearings with floating bushes, Author. dis. for a job. scientific step. Cand. tech. Sciences, Chelyabinsk (2011) (in Russian)

23. Sharma, A., Upadhyay, N., Kankar, P. K., Amarnath, M.: Nonlinear dynamic investigations on rolling element bearings: A review. Advances in Mechanical Engineering, 10(3), 1-15 (2018)

24. Kumar Vashisht, R, Peng, Q.: Nonlinear Dynamic Modeling of the Cracked Rotor Ball Bearing System With Emphasis on Damage Detection Capabilities. ASME. J. Vib. Acoust. 140(4), 041018-1-041018-10 (2018)

25. Li, Zigang, et al.: Nonlinear Dynamics of Unsymmetrical Rotor-Bearing System with Fault of Parallel Misalignment. Advances in Mechanical Engineering 10(5), 1-17 (2018)

26. Xu, Qi, et al.: Nonlinear Dynamic Behavior and Stability of a Rotor/Seal System with the Dynamic Vibration Absorber. Advances in Mechanical Engineering 11(1), 1-17 (2019) 
27. Nan, Guofang, et al.: Nonlinear Dynamic Mechanism of Rolling Element Bearings with an Internal Clearance in a Rotor-Bearing System. Advances in Mechanical Engineering 8(11), 19 (2016)

28. Ishida, Y., Liu, J.: Elimination of unstable ranges of rotors utilizing discontinuous spring characteristics: An asymmetrical shaft system, an asymmetrical rotor system, and a rotor system with liquid. Journal of Vibration and Acoustics, 132(1) (2010).

29. Xia, Z., Qiao, G., T. Zheng, W. Zhang.: Nonlinear modelling and dynamic analysis of the rotorbearing system. Nonlinear Dynamics 57, 559-577 (2009)

30. Luczko, J.: A geometrically nonlinear model of rotating shafts with internal resonance and self excited vibrations. Journal of Sound and Vibrations, 255(3), 433-456 (2002)

31. Penny, J., Friswell, M., Garvey, S., Lees, A.: Dynamics of rotating machines. - Cambridge University Press (2010)

32. Vance, J.M., Zeidan, F.Y. and Murphy, B.: Machinery vibration and rotordynamics. Wiley (2010)

33. Harris, T.A.: Rolling bearing analysis. 5-th edition. A Wiley-Interscience publication (2006)

34. David P.Fleming, Poplawski, J.V..: Transient vibration prediction for rotors on ball bearings using load-dependent non-linear bearing stiffness. NASA/TM-2002-211829.Glenn Research Center, Cleveland, Ohio (2002)

35. Lee, D.S., Choi, D.H.: A dynamic analysis of a flexible rotor in ball bearings with nonlinear stiffness characteristics. International Journal of Rotating Machinery, 3(2), 73-80 (1997)

36. Muszynska, A., Gran,t J.W.: Stability and instability of a two-mode rotor supported by two fluid-lubricated bearings. Journal of Vibration and Acoustics 113(3), 316-324 (1991)

37. Schweitzer, G., Maslen, E.H., Magnetic Bearings: Theory, Design, and Application to Rotating Machinery, Springer-Verlag Berlin Heidelberg (2009)

38. Xuening, Zh., Qinkai, H., Zhike, P., Fulei, Ch.: Stability analysis of a rotor-bearing system with time-varying bearing stiffness due to finite number of balls and unbalanced force. Journal of Sound and Vibration 332(25), 6768-6784 (2013)

39. Demailly, D., Throuverez, F., Jézéquel, L.: Unbalance responses of rotor/stator systems with nonlinear bearings by the time finite element method. International journal of rotating machinery 10(3), 155-162 (2004)

40. Greenheel, L.M.: Modeling of rolling element bearing mechanics. Contract NASA 8-38607. Monthly technical progress report. Sacramento, California (1991)

41. Duffing, G., Erzwungene Schwingungen bei veränderlicher Eigenfrequenz und ihre technische Bedeutung, Vieweg \& Sohn, Braunschweig (1918)

42. Shaw, S.W., Balachandran, B.: A review of nonlinear dynamics of mechanical systems in year 2008. Journal of System Design and Dynamics 2, 611-640 (2008)

43. Stoker, J.J.: Nonlinear Vibrations. Inter-science, New York (1950)

44. Rao, S.S.: Mechanical vibrations, Boston, MA: Addison Wesley, (2004)

45. Hayashi, Chihiro.: Nonlinear oscillations in physical systems. Princeton University Press (2014)

46. Tondl, A.: Domains of attraction for non-linear systems. National Res. Inst (1970) 
47. Nayfeh, A.H., Mook, D.: Nonlinear Oscillations. Wiley, New York (1979)

48. Benaroya, Haym, Mark Nagurka, and Seon Han.: Mechanical vibration: analysis, uncertainties, and control. CRC Press (2017)

49. Weaver, Jr, William, Stephen, Timoshenko, P., and Donovan Harold Young.; Vibration problems in engineering. John Wiley \& Sons (1990)

50. Klotter, K.: Nonlinear Vibration Problems Treated by the Averaging Method Of Ritz, W. Journal of Applied Mechanics-Transactions of the ASME. 18(3) (1951)

51. Magnus, Kurt, Karl Popp, and Walter Sextro. Schwingungen. Stuttgart: Teubner (1976)

52. Kovacic, Ivana, and Michael J. Brennan.; The Duffing equation: nonlinear oscillators and their behaviour. John Wiley \& Sons (2011)

53. Kovacic, Ivana, et al.: Jacobi elliptic functions: a review of nonlinear oscillatory application problems. Journal of Sound and Vibration 380, 1-36 (2016)

54. Kel'zon, A.S., Zhuravlev, Yu.N., Yanvarev, N.V.: Calculation and design of rotary machines. Engineering. Leningrad Direction (1977) (in Russian).

55. Kydyrbekuly, A., Khajiyeva, L., Gulama-Garyp, A. Y., Kaplunov, J.: Nonlinear Vibrations of a Rotor-Fluid-Foundation System Supported by Rolling Bearings. Strojniski Vestnik/Journal of Mechanical Engineering, 62(6), 351-362 (2016)

56. Hsu, C.S.: On the application of elliptic functions in nonlinear forced oscillations. Quarterly of Applied Mathematics 17, 393-407 (1960)

57. Starossek, U.: Exact analytical solutions for forced undamped Duffing oscillator. International Journal of Non-linear Mechanics. T. 85, 197-206 (2016)

58. Jin, Y., Lu, Z., Yang, R. et al.: A new nonlinear force model to replace the Hertzian contact model in a rigid-rotor ball bearing system. Appl. Math. Mech.-Engl. Ed. 39, 365-378 (2018) 
Figures
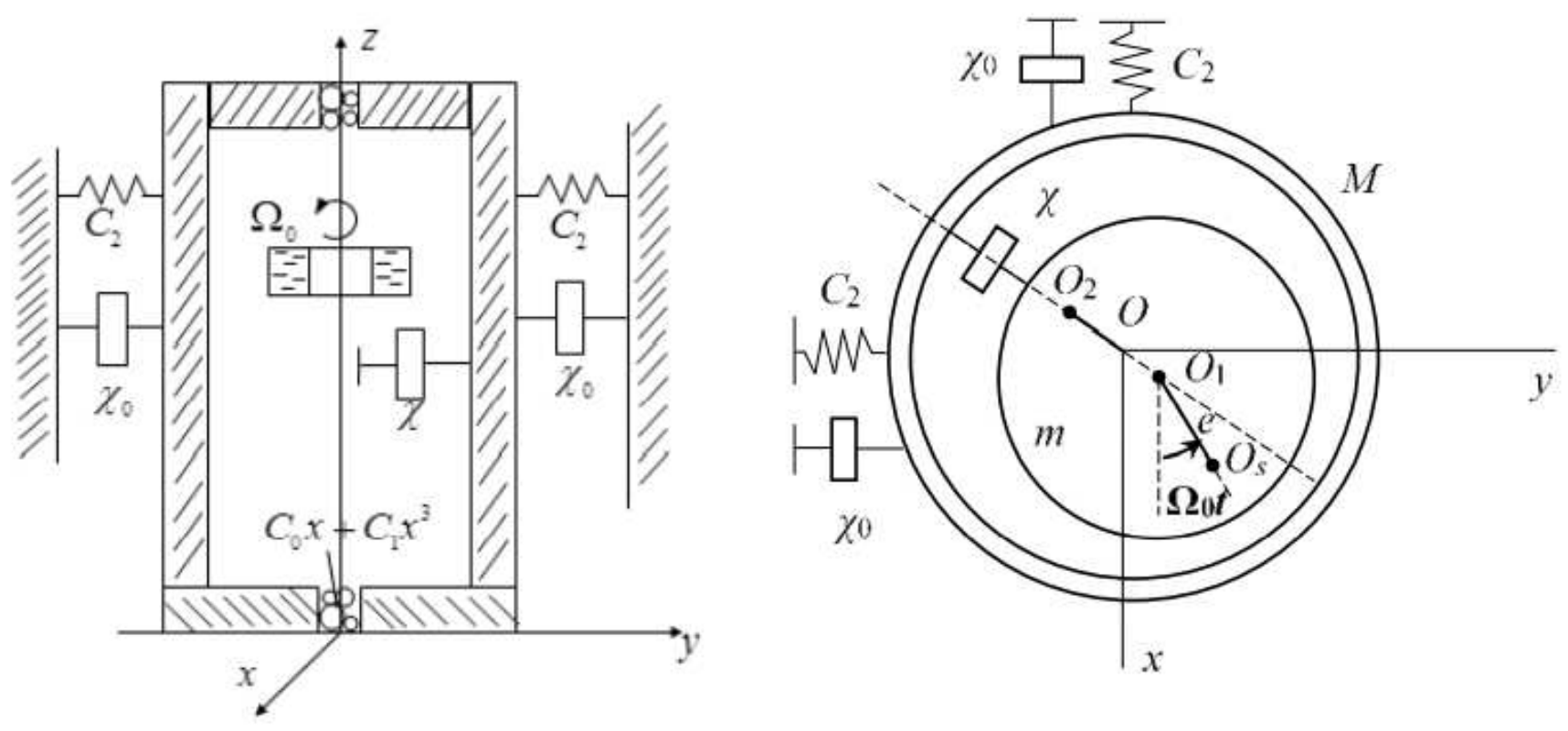

Figure 1

Scheme of a rotor on rolling bearings

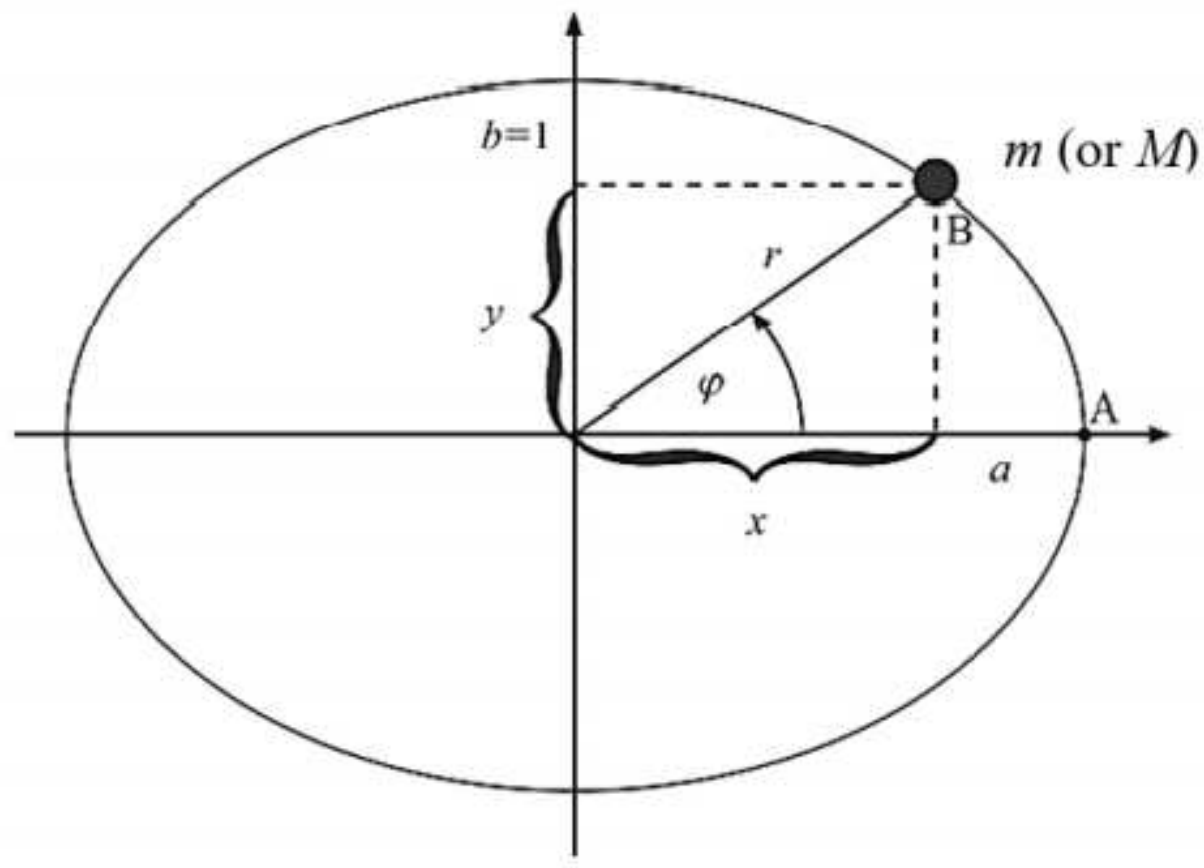

Figure 2

Interpretation the motion of the center of mass of the rotor and foundation 


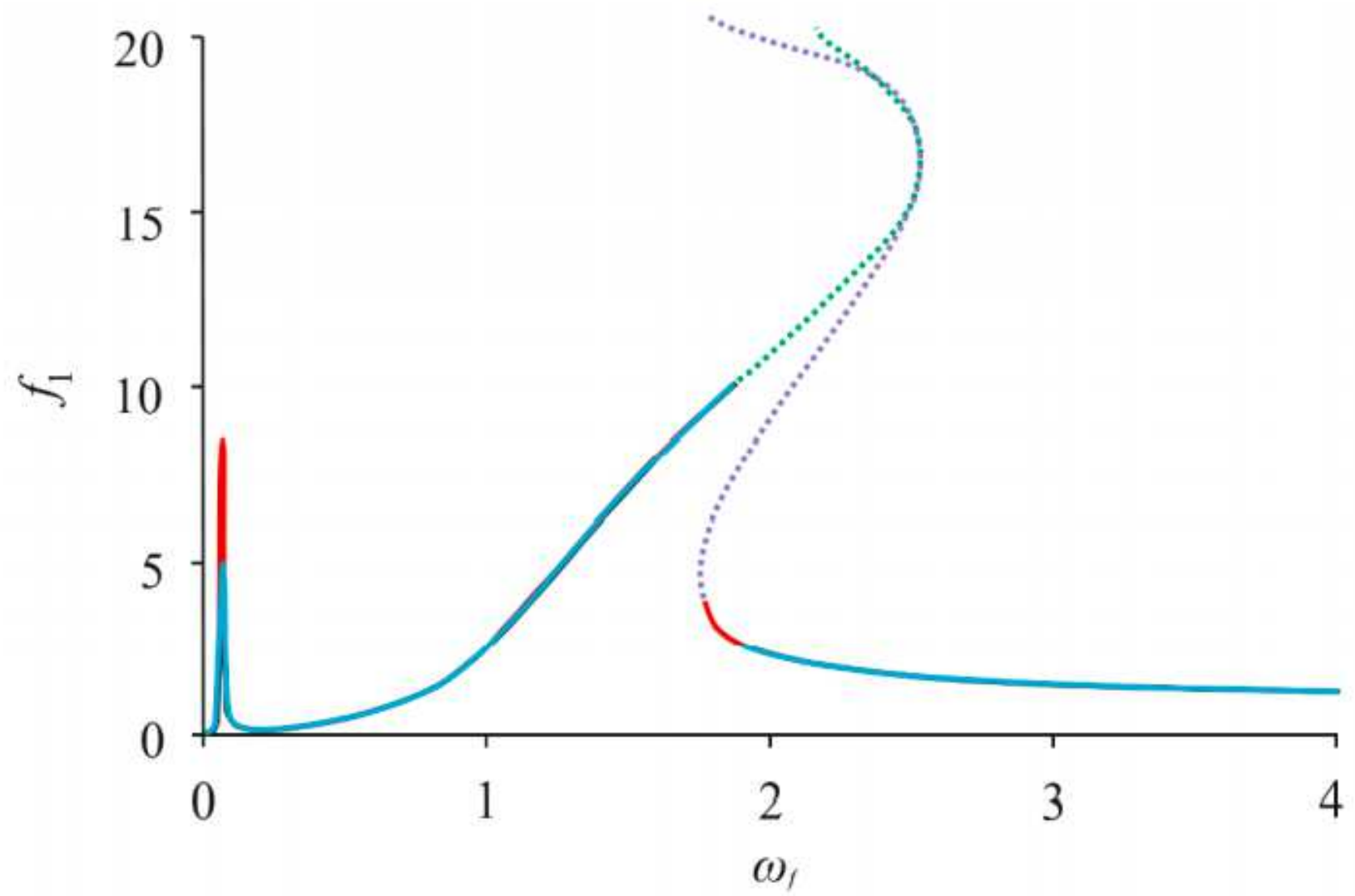

- Numerical Method

- Van der Pol's Method … EF Method

Figure 3

Frequency response of the rotor with different methods 


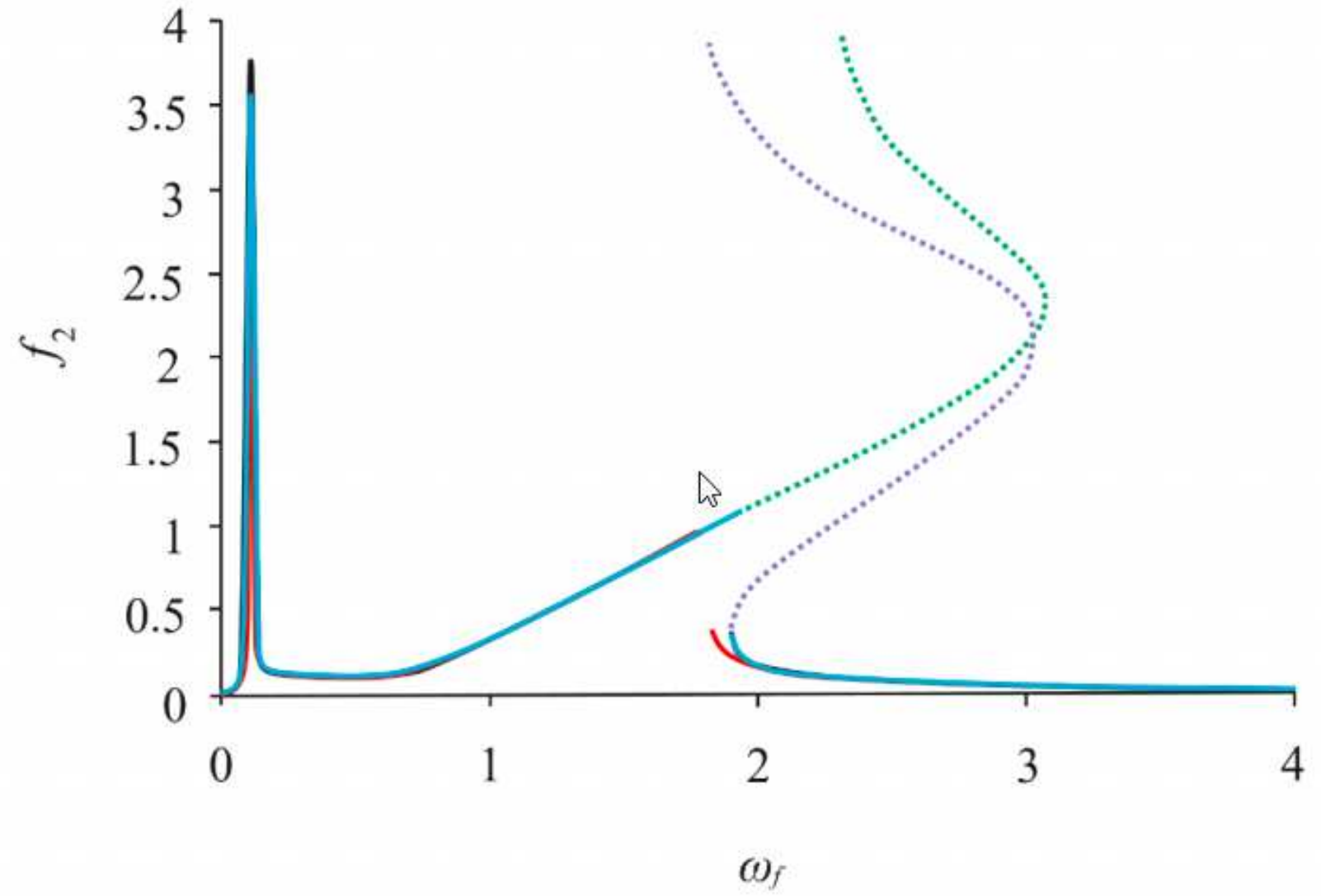

- Numerical Method - Van der Pol's Method … EF Method

Figure 4

Frequency response of the foundation using different methods 


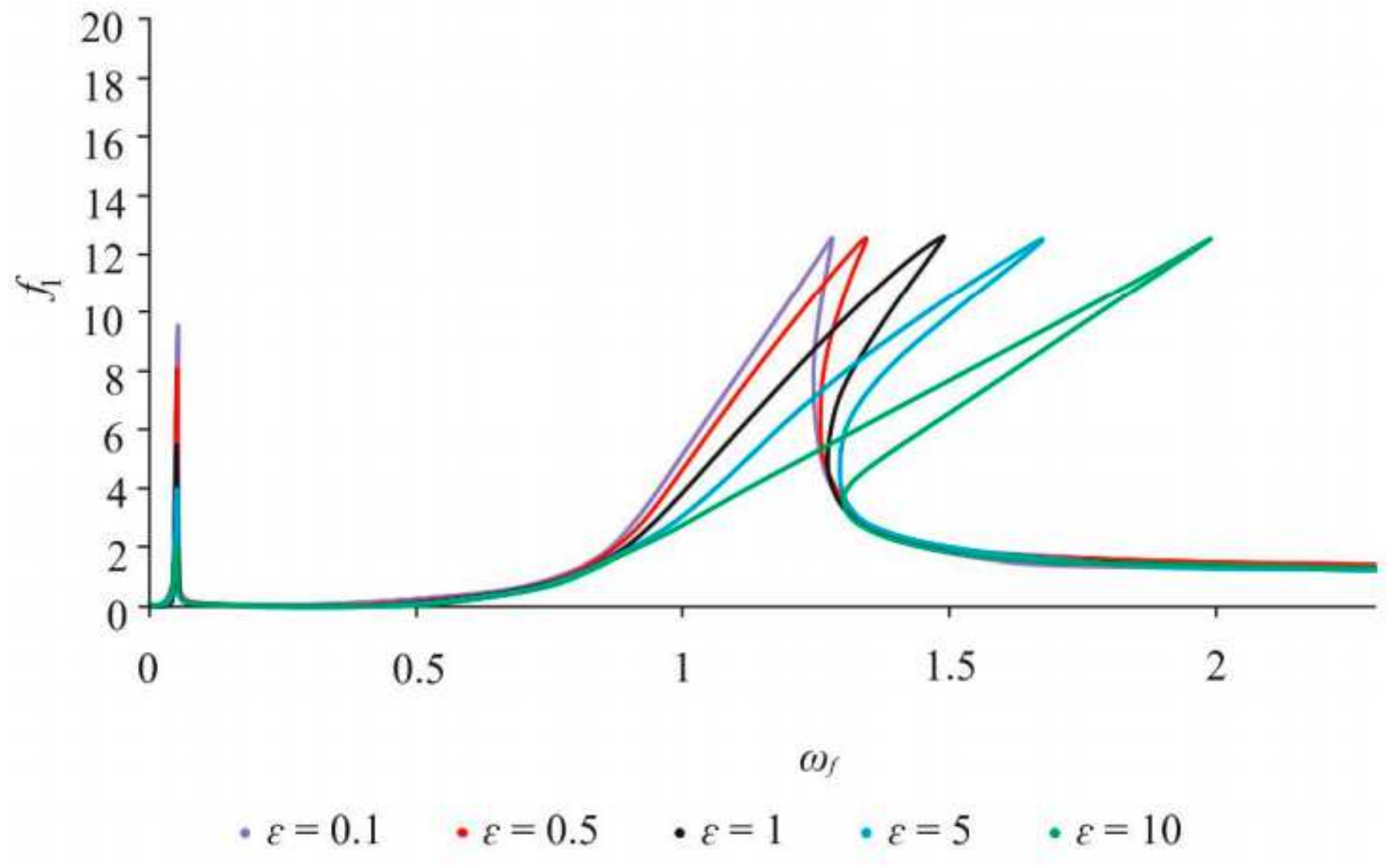

Figure 5

Frequency response of the rotor with different coefficients of nonlinearity 


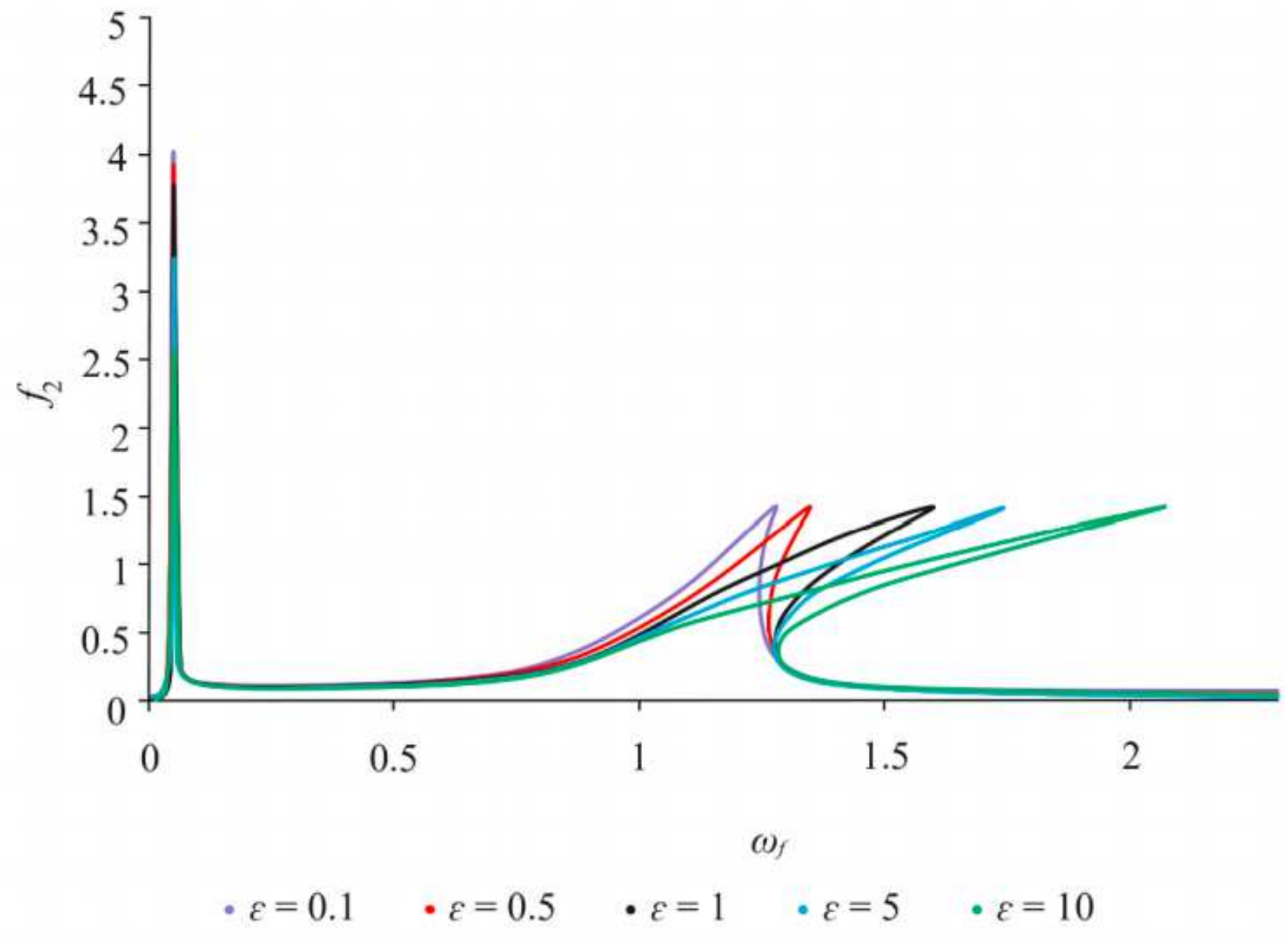

Figure 6

Frequency response of the foundation with different coefficients of nonlinearity 


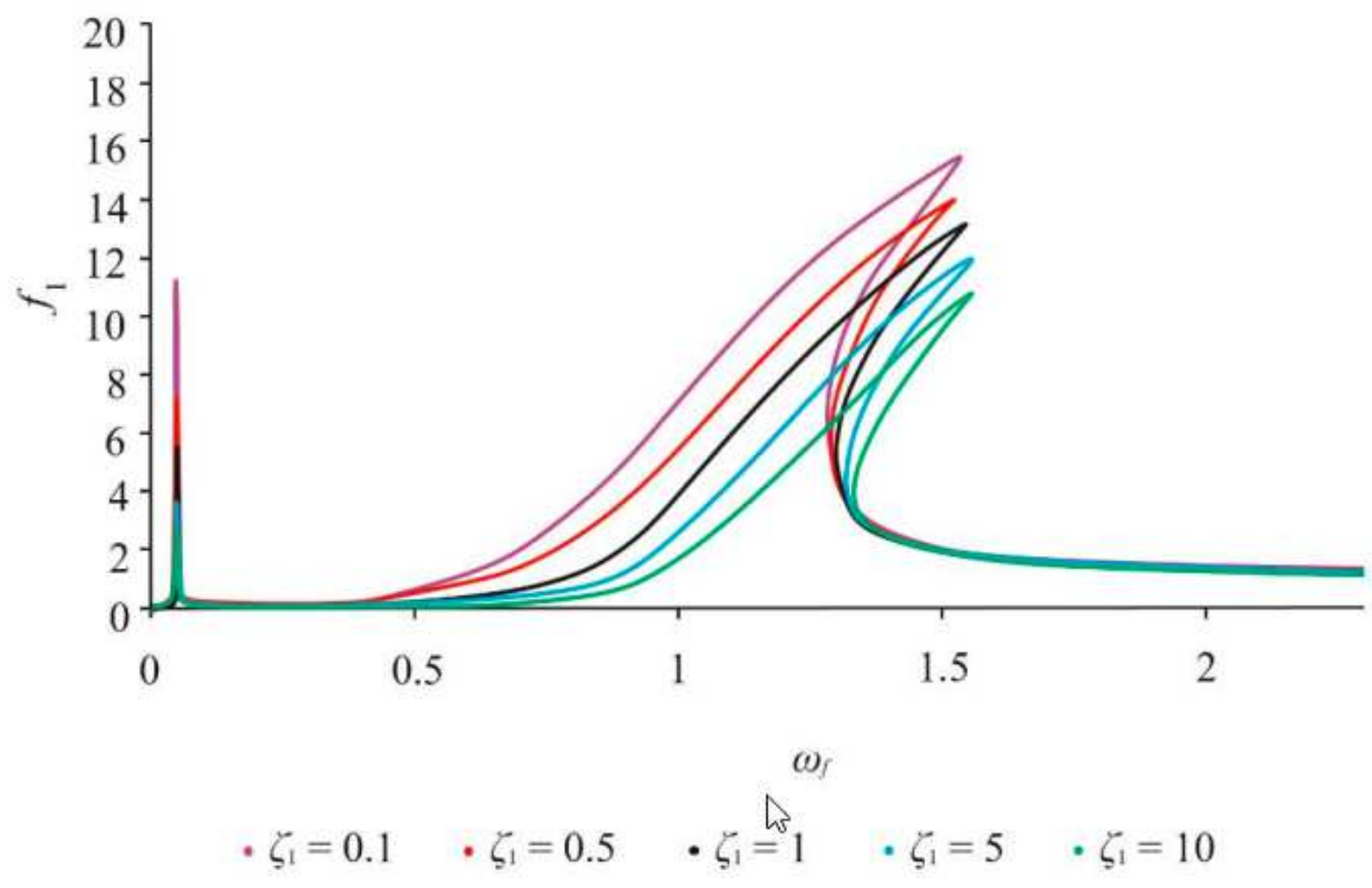

Figure 7

Frequency response of the rotor with different damping ratio 


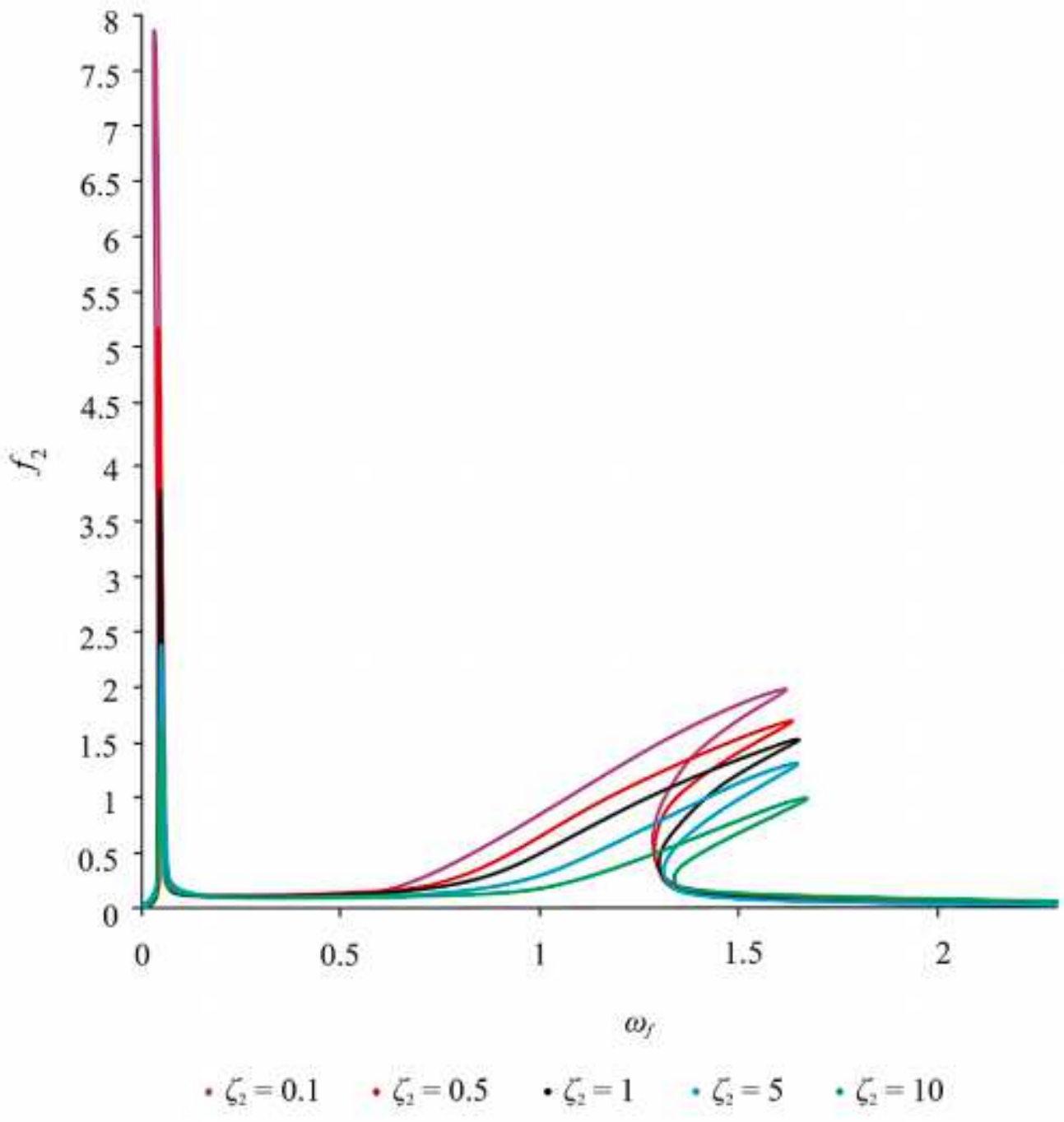

Figure 8

Frequency response of the foundation with different damping ratio 


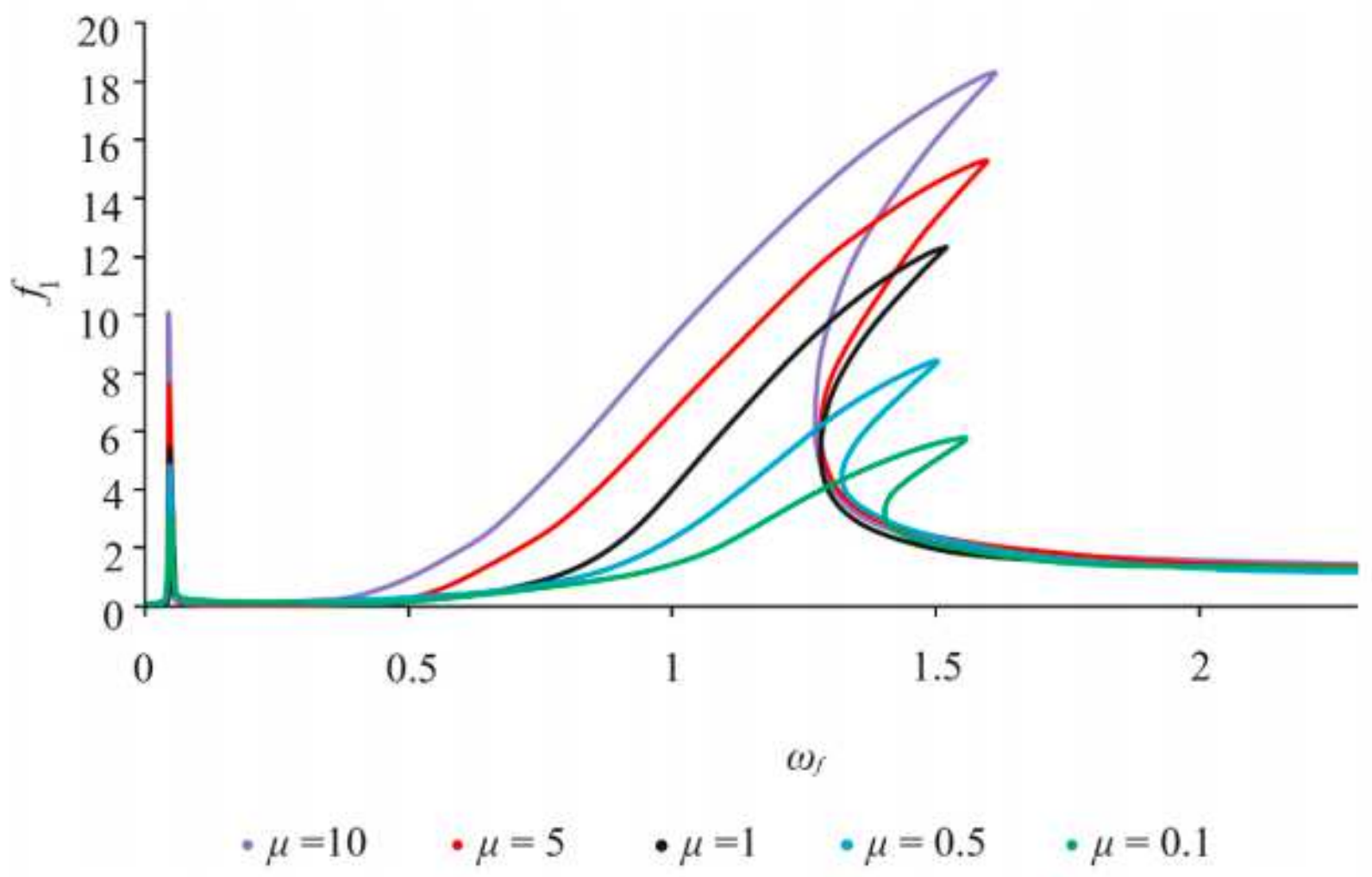

Figure 9

Frequency response of the rotor with different mass ratio 


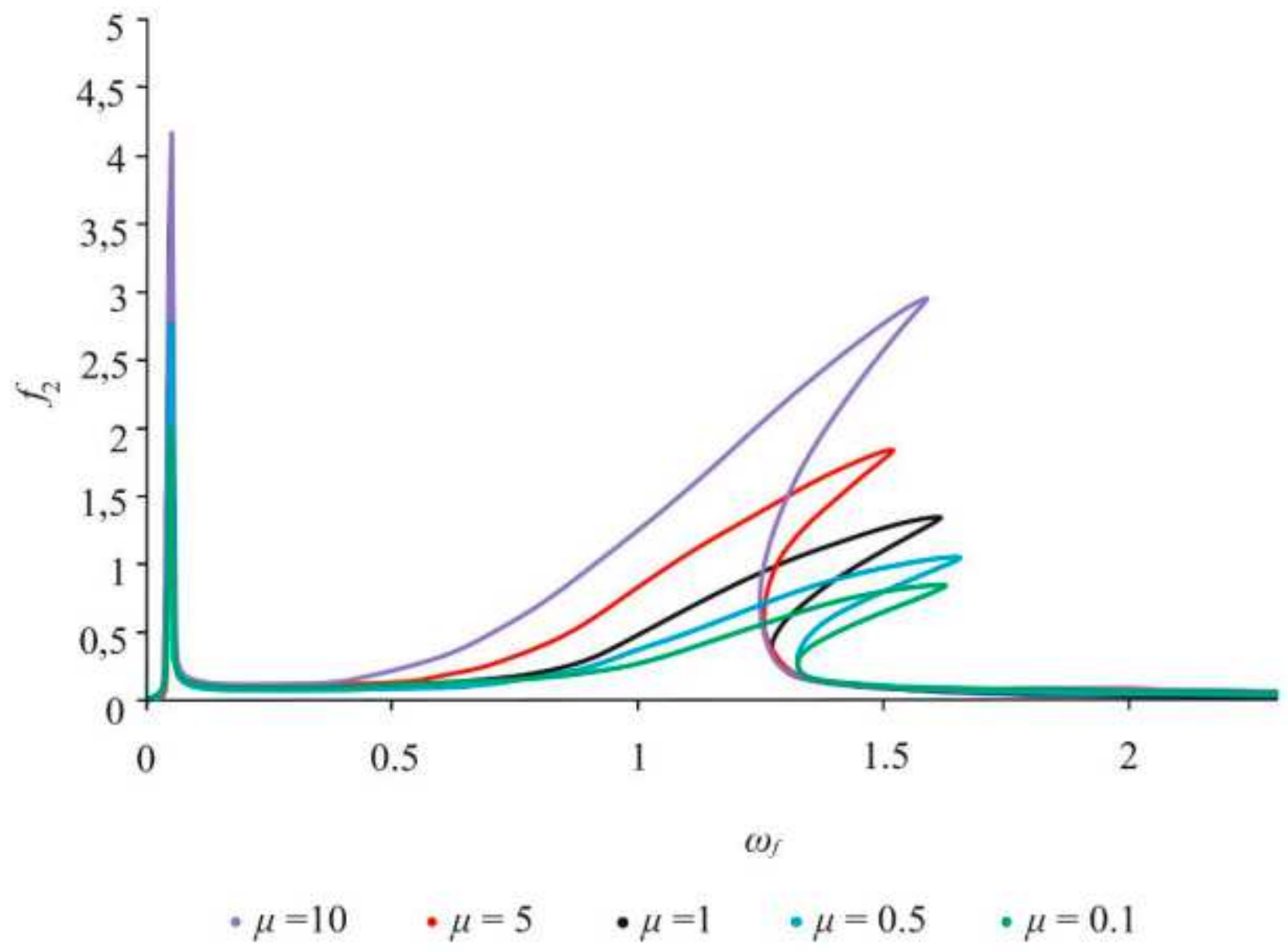

Figure 10

Frequency response of the foundation with different mass ratio

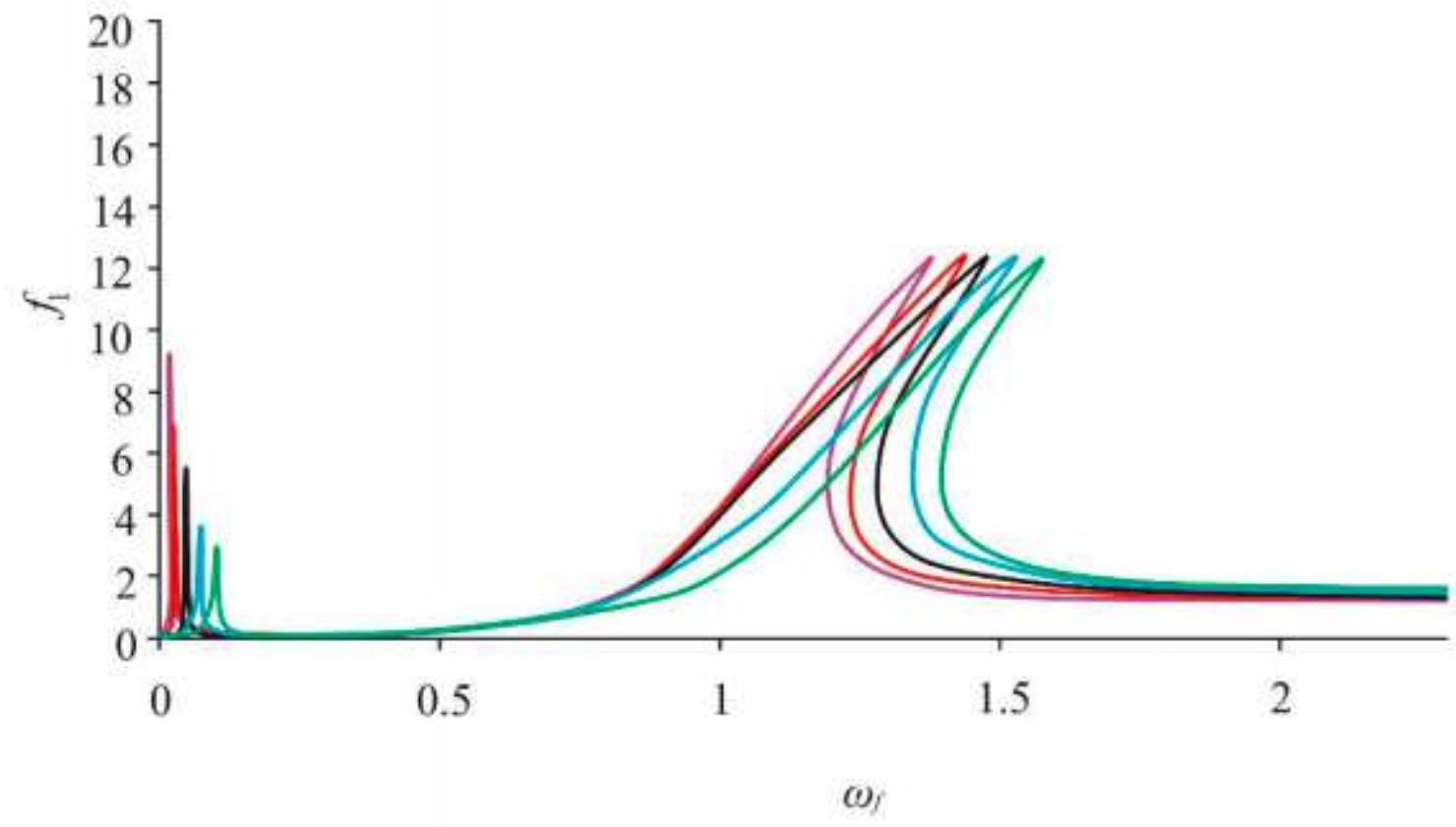

$$
\cdot \lambda=0.1 \quad \cdot \lambda=0.5 \quad \cdot \lambda=1 \quad \cdot \lambda=5 \quad \cdot \lambda=10
$$




\section{Figure 11}

Frequency response of the rotor with different stiffness ratio

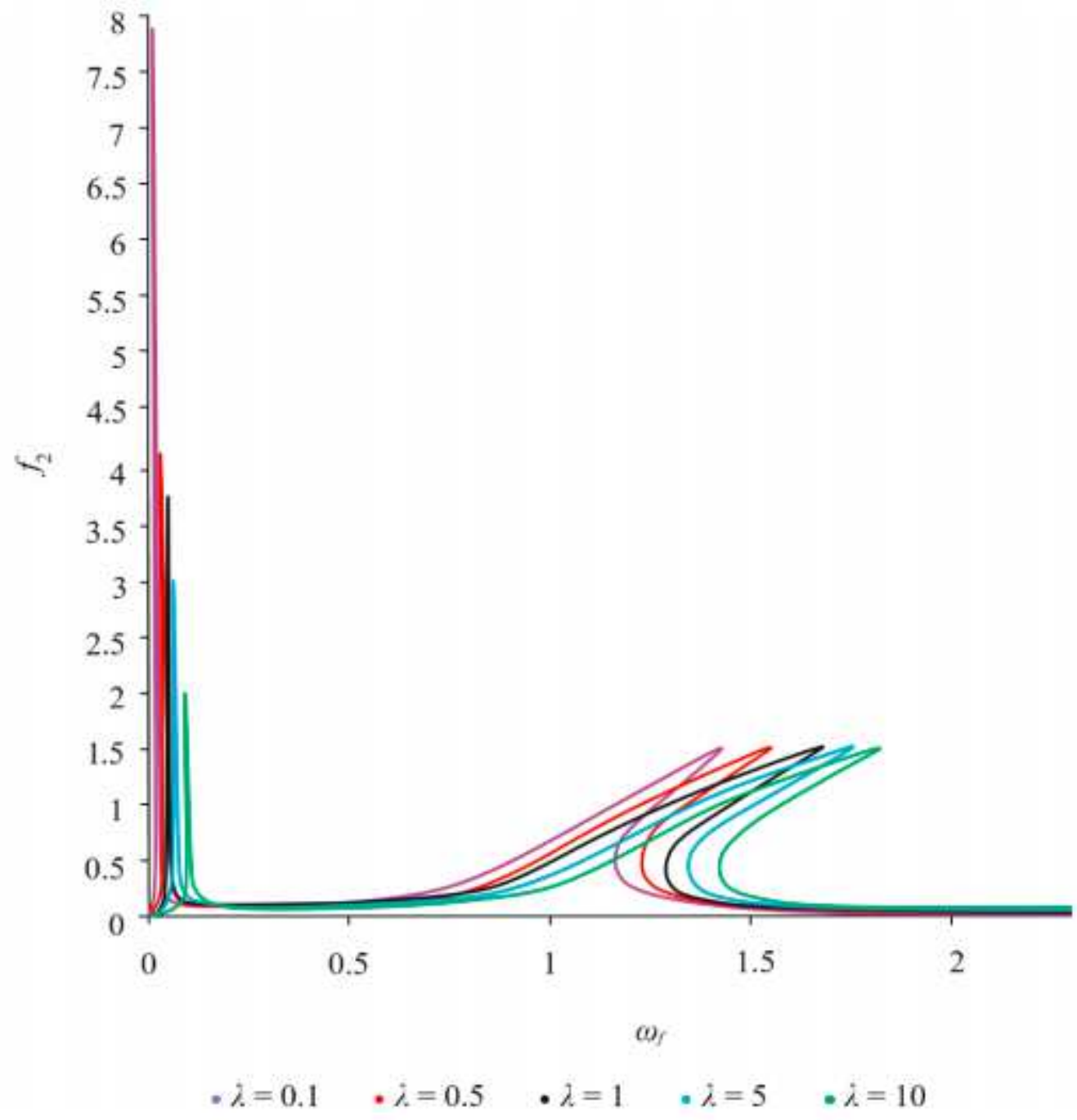

Figure 12

Frequency response of the foundation with different stiffness ratio 